\title{
Measurement and Classical Regime in Quantum Mechanics
}

\author{
Guido Bacciagaluppi \\ Department of Philosophy, University of Aberdeen \\ and \\ Institut d'Histoire et de Philosophie des Sciences et des Techniques \\ (CNRS, Paris 1, ENS), Paris*
}

28 August 2011

In this article, I shall focus on two of the main problems raising interpretational issues in quantum mechanics, namely the notorious measurement problem (discussed together with the theory of measurement in section 4) and the equally important but not quite as widely discussed problem of the classical regime (discussed together with decoherence in section 3). The two problems are distinct, but they are both intimately related to some of the issues arising from entanglement and density operators, which are thus briefly reviewed in section 2. A few fundamentals are rehearsed in section 1 . The article will aim to be fairly non-technical in language, but modern in outlook and covering the chosen topics in more depth than most introductory treatments.

The philosophy and foundations of quantum mechanics offer many more examples of live research issues, and much progress has been achieved recently in such traditional approaches as collapse theories, pilot-wave theories and Everett interpretations, and in the (time-honoured but recently revived) area of axiomatic reconstructions of the theory. Recent years have

*Address for correspondence: Department of Philosophy, University of Aberdeen, Old Brewery, High Street, Aberdeen AB24 3UB, Scotland, U.K. (e-mail: g.bacciagaluppi@abdn.ac.uk). 
seen fascinating advances also in the study of the other great puzzle raised by entanglement, namely quantum mechanical non-locality. No in-depth coverage of these other topics will be attempted.

\section{A few fundamentals}

\subsection{Phenomenology of measurements}

In classical mechanics, measurements are idealised as testing whether a system lies in a certain subset of its phase space. This can be done in principle without disturbing the system, and the result of the test is in principle fully determined by the state of the system. In quantum mechanics, none of these idealisations can be made. Instead: (i) measurements are idealised as testing whether the system lies in a certain (norm-closed) subspace of its Hilbert space $^{1}$ (ii) a measurement in general disturbs a system: more precisely (and in the ideal case), unless the state of the system is either contained in or orthogonal to the tested subspace, the state is projected onto either the tested subspace or its orthogonal complement (this is known as the 'collapse' of the quantum state, or the 'projection postulate'); (iii) this process is indeterministic, with a probability given by the squared norm of the projection of the state on the given subspace (the 'Born rule' or 'statistical algorithm' of quantum mechanics). ${ }^{2}$

For instance, take a spin- $1 / 2$ system initially in the state

$$
|\varphi\rangle=\alpha\left|+{ }_{x}\right\rangle+\beta\left|-{ }_{x}\right\rangle,
$$

where $\left|+_{x}\right\rangle$ and $\left|-_{x}\right\rangle$ are the states of $x$-spin up and down. If we test for $x$-spin-up (for the subspace $P_{+_{x}}$ ), the final state will be either $\left|+_{x}\right\rangle$ with probability $|\alpha|^{2}$, or $\left|-_{x}\right\rangle$ with probability $|\beta|^{2}$.

Often, one considers testing together a family of mutually orthogonal subspaces. ${ }^{3}$ Such a measurement is usually described as measuring a 'self-

\footnotetext{
${ }^{1} \mathrm{~A}$ subspace is a subset that is closed under linear combinations. We shall assume familiarity with the basic concepts of Hilbert spaces.

${ }^{2}$ Terminology varies, and sometimes the terms 'collapse postulate' or 'projection postulate' include also the Born rule.

${ }^{3}$ Note once and for all that we are not necessarily assuming that these subspaces are
} 
adjoint (linear) operator' (or 'observable')

$$
A=\sum a_{i} P_{i}
$$

where the (real) numbers $a_{i}$ are called the eigenvalues of the operator $A$, and are associated with the outcomes of the measurement. The $P_{i}$ are the projectors onto the given subspaces. ${ }^{4}$ These subspaces are called the eigenspaces of $A$, and are the subspaces of all vectors $\left|\psi_{i}\right\rangle$ (the eigenvectors of the operator) such that

$$
P_{i}|\psi\rangle=|\psi\rangle,
$$

or equivalently

$$
A|\psi\rangle=a_{i}\left|\psi_{i}\right\rangle \text {. }
$$

This is the origin of the traditional identification of quantum mechanical observables with (self-adjoint) operators. ${ }^{5}$

The collapse postulate then states that upon measurement of $A$ a state $|\psi\rangle$ will collapse onto $P_{i}|\psi\rangle$ (suitably renormalised), with probability $p_{i}=$ $\left\langle\psi\left|P_{i}\right| \psi\right\rangle$. The quantity

$$
\langle A\rangle_{\psi}:=\langle\psi|A| \psi\rangle=\left\langle\psi\left|\sum_{i} a_{i} P_{i}\right| \psi\right\rangle=\sum_{i} p_{i} a_{i}
$$

is then the average value or expectation value of the operator $A$ in the state $|\psi\rangle$. Note that unless the state is an eigenstate of the operator measured, there is a statistical spread of results, i.e. the dispersion of $A$ in the state $|\psi\rangle$,

$$
(\Delta A)_{\psi}:=\sqrt{\left\langle A^{2}\right\rangle_{\psi}-\langle A\rangle_{\psi}^{2}},
$$

one-dimensional. Alternatively, one can think of testing them in succession, in any order. Explicit application of the collapse postulate and the Born rule will show that one will obtain the same results with the same probabilities and the same final state, irrespectively of the order in which the tests are performed.

${ }^{4}$ Linear operators are mappings on the Hilbert space (or a subspace thereof) that map superpositions into the corresponding superpositions. The adjoint of a linear operator $A$ is a linear operator $A^{*}$ such that $\left\langle A^{*} \psi \mid \varphi\right\rangle=\langle\psi \mid A \varphi\rangle$ for all vectors $|\psi\rangle,|\varphi\rangle$ for which the two expressions are well-defined. An operator is self-adjoint iff $A=A^{*}$. A projection operator $P$ is a self-adjoint operator such that $P^{2}=P$. For ease of exposition, we shall mostly confine ourselves to the case of operators with 'discrete spectrum' (the sum in (2) is discrete), or even to finite-dimensional Hilbert spaces.

${ }^{5}$ Note that any self-adjoint operator can be decomposed uniquely into a sum (or more generally an integral) of projectors onto a family of mutually orthogonal subspaces. This is the so-called spectral theorem, which in elementary linear algebra is just the diagonalisability of self-adjoint matrices. 
is non-zero.

The association between self-adjoint operators and families of mutually compatible tests may seem purely conventional from the above description. This is not quite so. Self-adjoint operators play a further role in quantum mechanics, namely as (mathematical) generators of the unitary Schrödinger evolution. Now, think of a Stern-Gerlach spin experiment. A Stern-Gerlach magnet produces (approximately) a magnetic field that is inhomogeneous in just one spatial direction. Classically, what such a magnetic field can do is deflect along this direction a particle with non-zero magnetic moment, the amount of the deflection being proportional to the magnetic moment itself. In quantum mechanics, spin operators of the form

$$
S=\frac{\hbar}{2} P_{+}-\frac{\hbar}{2} P_{-}
$$

(with $P_{+}$and $P_{-}$the projection operators onto the 'up' and 'down' spin states in some direction) will appear in the Schrödinger evolution that couples the spin of the particle to its position degrees of freedom, and the deflection experienced by the particle will in fact be proportional to the eigenvalue $+\frac{\hbar}{2}$ or $-\frac{\hbar}{2}$. In this sense, the measurement is indeed sensitive to the eigenvalues of the corresponding spin operator, and not just to the projections of the state on the mutually orthogonal eigenspaces. ${ }^{6}$ This closer relation between a measurement and a single self-adjoint operator will be lost in the case of the generalised measurements discussed in section 4.4.

\subsection{Minimal interpretation and standard interpretation}

The above phenomenological rules yield a minimal interpretation of the formalism: some laboratory procedures are taken to be state preparations, and others are taken to be tests. Quantum mechanics yields probabilistic relations between states and outcomes of tests (Born rule). And, depending on

\footnotetext{
${ }^{6}$ Incidentally, note that whether a (classical or quantum) particle moves up or down in a Stern-Gerlach magnetic field will depend also on whether the inhomogeneous magnetic field is stronger at the north pole or at the south pole. Inverting either the gradient or the polarity of the field will invert the direction of deflection of a particle. (Since rotating the apparatus by 180 degrees corresponds to inverting both the gradient and the polarity, it has no net effect on the deflection.) Thus the choice of the words 'up' and 'down' for labelling the results is rather conventional. (The existence of these two different set-ups for measuring spin in the same direction is crucial in discussing contextuality and nonlocality in pilot-wave theory.)
} 
their outcome, tests are associated with further (preparatory) transformations of the state (collapse postulate). To be sure, the terms 'preparation' and 'test' (or 'measurement') are phenomenological, but in the cases in which we (or the working physicist) would normally apply them, any fundamental approach to quantum mechanics must allow us to recover the usual predictions of the theory, including in particular the fact that future predictions will depend on the previous outcomes in the way specified by the collapse postulate.

A common alternative interpretation of the formalism (often called the 'standard' or 'orthodox' or 'quantum logical' or 'Dirac-von Neumann' interpretation: we shall adopt the first of these terms) takes it that a quantum system has certain properties also independently of measurements, namely properties corresponding to tests that the system passes with probability 1 . These properties, which are uniquely fixed by the quantum state, can be further identified either with the state itself (or rather the one-dimensional subspace spanned by the vector state) - as is standardly done in the quantum logic literature, most explicitly by Jauch and Piron (1969) — or with an eigenvalue associated with that vector (hence also the name 'eigenstateeigenvalue link', due to Fine (1973), for this interpretational rule). ${ }^{7}$ For instance, an electron in a state of spin up in the $x$-direction will have a property corresponding to the vector $\left|+_{x}\right\rangle$, or, simply, a value $+\frac{\hbar}{2}$ for spin in the $x$-direction. According to the standard interpretation, a collapse of the quantum state is thus an actual change in the properties of the quantum system.

Assuming that quantum mechanics is meant to apply to any physical system whatsoever, and that there should not be a fundamental difference in the way it is interpreted across different domains, intuitions from the microscopic and the macroscopic domains of application of the theory will pull in different directions. Applying the minimal interpretation to macroscopic systems would mean that such systems will merely appear to have certain properties if measured (the Moon is not there until we look). In this domain, something like the standard interpretation would seem more natural

\footnotetext{
${ }^{7}$ Note that already according to the minimal interpretation, a quantum system described by a vector in Hilbert space has a set of dispositional properties to elicit specific responses with given probabilities in measurement situations (and these are fixed uniquely by the sure-fire disposition to elicit a certain response with probability 1 in a suitable measurement). The standard interpretation further identifies this set of dispositions with an intrinsic property of the system.
} 
(at least prima facie). On the other hand, applying the standard interpretation to the microscopic domain would mean that measurements appear to induce a discontinuous change in the properties of a microscopic system, in a way that is not necessarily compatible with the Schrödinger equation. This tension is the origin of the measurement problem of quantum mechanics (which we shall eventually discuss in section 4.6).

Obviously, the minimal interpretation is an instrumentalist interpretation, while the standard interpretation involves an ontological commitment to the quantum state. The former could be seen as a stripped-down version of some historically more accurate reading of the 'Copenhagen interpretation'. Note also that, while Schrödinger clearly had an ontological commitment to the wave function, it is not clear that it could be phrased in the abstract terms of the standard interpretation. He appears to have rather been interested in the 3-dimensional manifestation of his wave functions, in particular in terms of charge density (see also section 3 below). Something like the standard interpretation instead may have been adopted by both Dirac and von Neumann.

\section{Density operators and reduced states}

\subsection{Density operators}

Vectors in Hilbert space, as we have seen, define probability measures over the results of measurements of quantum mechanical observables. Indeed, up to phase factors, the association between unit vectors and such probability measures is one-to-one, since it is clear that if two unit vectors differ by other than an overall phase factor, there will be at least one test (the projection onto the subspace spanned by one of them), for which they will define different probabilities. ${ }^{8}$

To get rid of overall phase factors, we can also identify a quantum state defined by the vector $|\psi\rangle$ with the one-dimensional projection operator onto $|\psi\rangle$, denoted by $|\psi\rangle\langle\psi|$, i.e. the linear mapping that takes any vector state

\footnotetext{
${ }^{8}$ Note that thinking of Hilbert-space vectors in terms of their associated probability measures also makes readily intelligible why one considers only unit vectors. Indeed, normalisation of the vector ensures that the probabilities are correctly normalised, i.e. add up to 1 .
} 
$|\varphi\rangle$ to the state $\langle\psi \mid \varphi\rangle|\psi\rangle$ (the state $|\psi\rangle$ multiplied by the complex number $\langle\psi \mid \varphi\rangle)$. This can be suggestively written as

$$
|\psi\rangle\langle\psi|: \quad| \varphi\rangle \mapsto|\psi\rangle\langle\psi \mid \varphi\rangle
$$

This identification is particularly useful if one wishes to generalise the notion of a quantum state further. Indeed, it is clear that the probability measures defined by vectors in Hilbert space will not be the most general such probability measures. The set of these measures ought to be a convex set, i.e. closed under convex sums.

One can write a convex sum of two states corresponding to projection operators, say onto $\left|\psi_{1}\right\rangle$ and $\left|\psi_{2}\right\rangle$ as the operator

$$
\rho=p_{1}\left|\psi_{1}\right\rangle\left\langle\psi_{1}\left|+p_{2}\right| \psi_{2}\right\rangle\left\langle\psi_{2}\right|
$$

that maps any vector $|\varphi\rangle$ to the superposition

$$
p_{1}\left\langle\psi_{1} \mid \varphi\right\rangle\left|\psi_{1}\right\rangle+p_{2}\left\langle\psi_{2} \mid \varphi\right\rangle\left|\psi_{2}\right\rangle
$$

with $p_{1}+p_{2}=1$. We can now write the corresponding probability for the system passing a certain test represented by the projection $P$ as

$$
p_{\rho}(P)=\operatorname{Tr}(\rho P) \text {. }
$$

Here $\operatorname{Tr}(\rho P)$ is the symbol for the so-called trace of the operator $\rho P$, defined for any operator $A$ as

$$
\operatorname{Tr}(A)=\sum_{i}\left\langle\psi_{i}|A| \psi_{i}\right\rangle
$$

with the $\left|\psi_{i}\right\rangle$ forming a basis of the Hilbert space. ${ }^{9}$

As already mentioned in section 1.1, operators of the form $A|\psi\rangle=a_{i}\left|\psi_{i}\right\rangle$ can be used to classify simultaneous experimental tests for families of mutually orthogonal subspaces. A system will test positively to only one of these tests, and to this test can be associated an eigenvalue of the corresponding operator. Since $\operatorname{Tr}\left(\rho P_{i}\right)$ is the probability for the outcome $i$ in a test of $P_{i}$, the expression

$$
\operatorname{Tr}(\rho A)=\sum a_{i} \operatorname{Tr}\left(\rho P_{i}\right)
$$

\footnotetext{
${ }^{9}$ One can check that the definition of the trace is indeed independent of the basis. In finite dimensions and given a matrix representation of $A$, the trace is simply the sum of the diagonal elements of the corresponding matrix.
} 
is equal to the expectation value of the self-adjoint operator $A$.

The operator $\rho$ is known as a density operator, because in the expression (11) it plays a role similar to that of a probability density. Note that the onedimensional projection operators are the extremal elements of the convex set of density operators, those that cannot be decomposed further in terms of convex combinations of other density operators.

Now, it is a deep theorem due to Gleason (1957) that the states defined by density operators are the most general probability measures that can be defined over the possible tests that can be (ideally) performed on a quantum system. A probability measure in Gleason's sense, as one would expect, is a positive, normalised mapping that in the finite-dimensional case is additive and in the infinite-dimensional case $\sigma$-additive for families of mutually orthogonal projectors. ${ }^{10}$

Quantum mechanical states in the sense of density operators can be alternatively characterised as the most general (linear) expectation value functionals on the self-adjoint operators. This is actually what von Neumann shows in what has come to be known as his no-hidden-variables theorem (von Neumann 1932, pp. 305-24 of the English translation). More precisely, von Neumann takes a state $s$ to be an assignment of an expectation value to each self-adjoint operator $A$, subject to a continuity requirement (which is vacuous in finite dimensions), a trivial normalisation requirement $s(\mathbf{1})=1$, and a linearity requirement

$$
s(A+B)=s(A)+s(B)
$$

for any two observables $A$ and $B$. He then proves that the only such expectation functionals on the self-adjoint operators are of the form $\operatorname{Tr}(\rho A)$, with $\rho$ a density operator. That is, the most general states in this sense are indeed the quantum mechanical states.

Von Neumann took this result as showing that there could be no more precise description of ensembles of quantum mechanical systems (in particular no states with zero dispersion for all observables), and thus as ruling out 'hidden variables'. Note, however, that von Neumann himself explicitly points out that assumption (14) is natural in the context of commuting

\footnotetext{
${ }^{10}$ Normalisation means $p(\mathbf{1})=1$, with $\mathbf{1}$ the identity operator, i.e. the projection onto the whole of the Hilbert space. The theorem holds for quantum systems with Hilbert space of dimension at least 3 (but see the remark at the end of section 4.4 below).
} 
observables (where we see it is analogous to Gleason's additivity requirement), but is a very non-trivial assumption in the case of non-commuting ones (pp. 308-9). As noted forcibly by Grete Hermann (1935), this vitiates his conclusion about hidden variables. ${ }^{11}$

A very simple geometrical intuition for the convex structure of density operators in the case of spin-1/2 systems can be gained as follows. Imagine mapping each state of spin-up in the direction $\mathbf{r}$ to the corresponding unit vector in three spatial dimensions,

$$
\mathbf{r}=\left(\begin{array}{l}
r_{x} \\
r_{y} \\
r_{z}
\end{array}\right)
$$

This mapping between the vector states of a spin- $1 / 2$ system and the unit sphere is a bijection (one-to-one and onto). It turns out that it can be extended to an affine isomorphism, i.e. a map that preserves convex combinations. What this means in particular is that for any two vector states $|\psi\rangle$ and $|\varphi\rangle$, which are mapped onto unit vectors $\mathbf{r}$ and $\mathbf{s}$ on the sphere, we can map the density operator

$$
\rho=\lambda|\psi\rangle\langle\psi|+(1-\lambda)| \varphi\rangle\langle\varphi|
$$

to the point $\lambda \mathbf{r}+(1-\lambda) \mathbf{s}$ in the interior of the unit ball in three dimensions.

This representation is known as the Bloch sphere or the Poincaré sphere. We can use it to establish geometrically many propositions about density operators. Here are a few examples. Density operators can be decomposed non-uniquely as convex combinations of vector states, in fact in infinitely many ways, and as combinations of arbitrarily many vector states (even continuously many). On the other hand, for each density operator, there is generally a unique decomposition as a combination of spin-up and spindown in a single direction (as a combination of antipodal points on the

\footnotetext{
${ }^{11}$ The relevant section 7 in Hermann's essay has been translated into English by M. Seevinck (see http://mpseevinck.ruhosting.nl/seevinck/trans.pdf). The same point was famously made by Bell (1966), who further pointed out the absurdity of requiring linearity of the hypothetical 'dispersion-free states' (which would have to assign an eigenvalue to each observable as a definite value). Bell uses the following example: consider the operators $\sigma_{x}, \sigma_{y}$ and $\sigma_{x}+\sigma_{y}$. For a linear, dispersion-free state $\lambda$,

$$
\left\langle\sigma_{x}+\sigma_{y}\right\rangle_{\lambda}=\left\langle\sigma_{x}\right\rangle_{\lambda}+\left\langle\sigma_{y}\right\rangle_{\lambda}
$$

But the left-hand side takes the possible values $\pm \sqrt{2}$, while the right-hand side takes the possible values $-2,0,+2$, so that (15) cannot be satisfied.
} 
sphere). ${ }^{12}$ The only exception is the state that lies at the centre of the ball, which is the equal-weight combination of up and down states in any direction ('maximally mixed' state). We also see that the only states that are extremal (also called pure states) in the convex set of density operators are indeed the vector states that map to the unit vectors on the sphere.

\section{$2.2 \quad$ Proper and improper mixtures}

The non-uniqueness in general of convex decompositions of a density operator is one of their most striking features, and a major difference between probability measures in quantum and classical mechanics.

Also in classical mechanics one can introduce states that are convex combinations of the pure states defined by points in the phase space (which correspond to trivial — or 'dispersion-free' — probability distributions). These general states are simply probability measures over phase space. But it is always possible to decompose a classical probability measure uniquely as a convex combination of extremal states (a convex set with this property is known as a 'simplex'). Indeed, both mathematically and physically, when we deal with a probabilistic state in classical mechanics, we are always dealing with a statistical mixture of non-probabilistic states, i.e. probabilities arise through our ignorance of the actual pure state of the system, and any statistical distributions of measurement results are attributable to this same ignorance. There is no possible ambiguity, since the space of classical probability measures is a simplex.

In quantum mechanics, things are different. Even though formally density operators can always be written as 'mixtures', i.e. as convex combinations of pure states, at the very least their non-unique decomposability will introduce an ambiguity in their interpretation. Assuming that in some case a density operator has arisen through our ignorance of the actual pure state of the system, this is not manifest in the form of the density operator. We might know that the spread of results observed in our tests is partly due to our ignorance of what the quantum state actually is, and partly due to the probabilistic nature of the vector states themselves, but knowledge of how to thus 'apportion the blame' is knowledge in excess of that encoded in

\footnotetext{
${ }^{12}$ Technically, a density operator (in arbitrary dimensions) is a 'compact operator', and for such operators a discrete (if not necessarily finite) decomposition as a sum of mutually orthogonal projectors always exists.
} 
the density operator itself. It corresponds formally not just to the density, but to a particular convex decomposition. Unlike the classical case, this decomposition cannot be uniquely retrieved from the state alone.

This feature of quantum mechanical 'mixtures' is essential to the question of how they should be understood, especially in the context of our distinction between the minimal and standard interpretations of the theory. There is, however, an even more essential issue for the question of how to understand density operators. Of course, density operators can arise as genuine statistical mixtures of pure quantum states (for instance a state obtained by randomly mixing systems prepared in different pure states). This is generally referred to as a proper mixture. So, for instance, if we know that a measurement of spin- $x$ on an electron has been actually carried out, but we are ignorant of the result, then we should apply the collapse postulate, but average over the results (so-called non-selective measurement). In this case we will have a proper mixture of the states $\left|+_{x}\right\rangle$ and $\left|-{ }_{x}\right\rangle$ due to ignorance (we do not know which state we should actually best use for further predictions). ${ }^{13}$

However, there are other cases in which density operators arise that are not thus related to our ignorance, namely as so-called reduced states, states of subsystems of a larger system described by an entangled pure state.

Indeed, the phenomenological rules sketched in section 1.1 (collapse postulate and Born rule) turn out to have surprising consequences when applied to the case of entangled states. Take a singlet state of two spin- $1 / 2$ systems

$$
\frac{1}{\sqrt{2}}\left(\left|+{ }_{x}^{1}\right\rangle \otimes\left|-{ }_{x}^{2}\right\rangle-\left|-{ }_{x}^{1}\right\rangle \otimes\left|+{ }_{x}^{2}\right\rangle\right)
$$

and test for $P_{+_{x}}^{1} \otimes P_{+_{x}}^{2}$. The test will come out negative with probability 1 , and the state will be undisturbed, since it lies in a subspace orthogonal to the tested one. Now test for $P_{+_{x}}^{1} \otimes P_{-x}^{2}$. The result will be $\left|+{ }_{x}^{1}\right\rangle \otimes\left|-{ }_{x}^{2}\right\rangle$ or $\left|-{ }_{x}^{1}\right\rangle \otimes\left|+{ }_{x}^{2}\right\rangle$, each with probability $1 / 2$. In this case, we see that the results of the spin measurements performed on the two electrons are perfectly (anti-)correlated. Correlations, albeit weaker, will be observed quite in general if spin is measured along two different directions on the two subsystems

\footnotetext{
${ }^{13}$ Of course the collapse postulate is a phenomenological rule, so if one does not believe that collapse is fundamental, there is a sense in which proper mixtures cannot be prepared in this way. Nevertheless, any fundamental approach to quantum mechanics, even if it denies the reality of collapse, will have to explain the appearance of the possibility of preparing proper mixtures, just as it will have to explain the appearance of collapse.
} 
(as can be easily checked explicitly). Entanglement thus introduces what appear to be irreducible correlations between results of measurements (even carried out at a distance), and this for a generic pair of tests. This is the origin of nonlocality in quantum mechanics.

On the other hand, performing a measurement (or any other manipulation) on one of a pair of entangled particles does not affect the probability distributions for results of measurements on the other. This is the so-called no-signalling theorem. (That is, while conditionalising on the outcomes of one measurement in general affects the probabilities for the other, conditionalising on performing the measurement does not.) It is easy to see this in the example: we have perfect anti-correlations between outcomes on the two sides, but averaging over the outcomes on one side yields back the usual 50-50 distribution on the other side. By explicit calculation, one can check the claim in the general case, i.e. for measurements along different spin directions on the two sides.

The no-signalling theorem is crucial to our purposes, since it allows us to generalise the description of quantum systems to subsystems of entangled systems. Indeed, although such subsystems cannot be associated with any vector in their Hilbert space, we can assign them a suitable probability measure for each test we may want to carry out on them, because the nosignalling result guarantees that the probability of such a test is well-defined independently of whether any test (or which one) is carried out on the rest of the system. So, we can define a probability measure for a test on a subsystem by simply taking the marginal of the probability measure associated with the entangled state of the total system when the relevant test is paired with an arbitrary test on the rest of the system. But now, because of Gleason's theorem, we know that such a state must be given by a density operator.

Let us see this in a concrete example. Suppose we wish to define the probability for a measurement of $\operatorname{spin}-x$ on one of a pair of spin- $1 / 2$ systems in some arbitrary entangled state. We can write the state of the pair as

$$
\alpha_{++}\left|+{ }_{x}^{1}\right\rangle \otimes\left|+{ }_{x}^{2}\right\rangle+\alpha_{+-}\left|+{ }_{x}^{1}\right\rangle \otimes\left|-{ }_{x}^{2}\right\rangle+\alpha_{-+}\left|-{ }_{x}^{1}\right\rangle \otimes\left|+{ }_{x}^{2}\right\rangle+\alpha_{--}\left|-{ }_{x}^{1}\right\rangle \otimes\left|-{ }_{x}^{2}\right\rangle .
$$

If we were to measure spin- $x$ on both electrons of the pair, the resulting Born-rule probabilities would be

$$
p_{++}=\left|\alpha_{++}\right|^{2} \quad p_{+-}=\left|\alpha_{+-}\right|^{2} \quad p_{-+}=\left|\alpha_{-+}\right|^{2} \quad p_{--}=\left|\alpha_{--}\right|^{2},
$$


and averaging over the results for the second electron, we obtain

$$
p_{+}=\left|\alpha_{++}\right|^{2}+\left|\alpha_{+-}\right|^{2} \quad \text { and } \quad p_{-}=\left|\alpha_{-+}\right|^{2}+\left|\alpha_{--}\right|^{2} .
$$

In this way, one can determine the probabilities for arbitrary tests on the first (and similarly on the second) electron, and so associate with it a state in Gleason's sense (a probability measure for any family of mutually orthogonal projections), even though it is not described by a vector in Hilbert space.

A more compact way of thinking of such a state is in terms of a convex combination of the states that one would obtain through the collapse postulate were one to perform a measurement on the other electron. So, for instance, if one were to perform a measurement of spin- $x$ on the first electron, one would obtain the two (normalised) states:

$\frac{1}{p_{+}}\left(\alpha_{++}\left|+{ }_{x}^{1}\right\rangle \otimes\left|+{ }_{x}^{2}\right\rangle+\alpha_{+-}\left|+{ }_{x}^{1}\right\rangle \otimes\left|-{ }_{x}^{2}\right\rangle\right)=\frac{1}{p_{+}}\left|+{ }_{x}^{1}\right\rangle \otimes\left(\alpha_{++} \otimes\left|+{ }_{x}^{2}\right\rangle+\alpha_{+-}\left|-_{x}^{2}\right\rangle\right)$

and

$\frac{1}{p_{-}}\left(\alpha_{-+}\left|-{ }_{x}^{1}\right\rangle \otimes\left|+{ }_{x}^{2}\right\rangle+\alpha_{--}\left|-_{x}^{1}\right\rangle \otimes\left|-{ }_{x}^{2}\right\rangle\right)=\frac{1}{p_{-}}\left|{ }_{x}^{1}\right\rangle \otimes\left(\alpha_{-+} \otimes\left|+{ }_{x}^{2}\right\rangle+\alpha_{--}\left|-{ }_{x}^{2}\right\rangle\right)$.

Writing

$$
\left|\psi_{+}\right\rangle=\alpha_{++} \otimes\left|+{ }_{x}^{2}\right\rangle+\alpha_{+-}\left|-_{x}^{2}\right\rangle
$$

and

$$
\left|\psi_{-}\right\rangle=\alpha_{-+} \otimes\left|+_{x}^{2}\right\rangle+\alpha_{--}\left|-_{x}^{2}\right\rangle,
$$

we see that the state of the second electron would collapse to $\left|\psi_{+}\right\rangle$or $\left|\psi_{-}\right\rangle$ with the probabilities $p_{+}$and $p_{-}$(defined by (21)), respectively.

We can now determine the probabilities for any tests on the second electron by taking the weighted average of the probabilities defined by $\left|\psi_{+}\right\rangle$ and $\left|\psi_{-}\right\rangle$, with the weights $p_{+}$and $p_{-}$, respectively. We call this the reduced state of the second electron, and write it formally as

$$
\rho=p_{+}\left|\psi_{+}\right\rangle\left\langle\psi_{+}\left|+p_{-}\right| \psi_{-}\right\rangle\left\langle\psi_{-}\right| \text {. }
$$

This representation makes it explicit that a reduced state is a density operator. Furthermore, the no-signalling theorem shows us explicitly that the representation (26) cannot be unique. If a different measurement were to be carried out on the first electron, then the states (24) and (25) would 
have to be different, if the total state is entangled, and the corresponding probabilities would generally also be different. As a simple example, take the singlet state (18). Measuring spin in direction $\mathbf{r}$ on the first electron will collapse the second electron into a state of spin in the same direction $\mathbf{r}$, whatever this might be, due to the rotational symmetry of the state. Thus, the reduced state of an electron from a pair in the singlet state will have the form

$$
\rho=\frac{1}{2}\left|+_{\mathbf{r}}\right\rangle\left\langle+_{\mathbf{r}}\left|+\frac{1}{2}\right|-_{\mathbf{r}}\right\rangle\left\langle-_{\mathbf{r}}\right|,
$$

(in the case of the singlet the probabilities for the different results will always be equal to $1 / 2$ ) and will be independent of $\mathbf{r} .^{14}$

How are we to interpret density operators arising as reduced states of entangled systems? Certainly not as proper mixtures! Indeed, if a composite quantum system is in a pure entangled state, this state cannot be further decomposed as a weighted average of other quantum states, so cannot be interpreted in terms of ignorance. But then, neither can the states of the subsystems be interpreted in terms of ignorance, despite the fact that the subsystems are necessarily described by density operators. Contrapositively, were the subsystems themselves in pure states (and we ignorant of which pure states they were in), then the composite would be in a mixed state, because it would actually be in a product state (but we ignorant of which product state it was in).

A mixed state arising as the reduced state of a subsystem, where the total system is in a pure state, is generally referred to as an improper mixture. The reduced state of an electron from an entangled pair is a paradigm example of an improper mixture, so that a decomposition such as (26) should not be taken as indicating that the system is indeed either in the state $\left|\psi_{+}\right\rangle$or in the state $\left|\psi_{-}\right\rangle$.

At least from the point of view of the minimal interpretation, there is nothing especially problematic about this. Quantum systems have dispositional properties to elicit certain outcomes under certain test circumstances, irrespectively of whether we seek to explain them further. If we do seek to explain these further, the case of subsystems of entangled systems will turn out to be particularly tricky, but from the point of view of the minimal interpretation it is perfectly natural for subsystems of entangled systems to have such dispositional properties. The only aspect of note is that in the

\footnotetext{
${ }^{14}$ Geometrically, this is the maximally mixed state at the centre of the Bloch sphere.
} 
case of such subsystems we explain the distributions over outcomes purely in dispositional terms (just as in the case of systems in pure states), while in other cases, we may have reason to analyse the distributions over outcomes partially in terms of ignorance.

Instead, the existence of entanglement and reduced states has rather disquieting consequences for the standard interpretation. Indeed, if the system is neither in the state $\left|\psi_{+}\right\rangle$nor in the state $\left|\psi_{-}\right\rangle$(nor in any other state that might appear in a convex decomposition of the density operator of the system), then the system simply lacks any of the properties that in the standard interpretation are associated with these states. We can still apply the standard interpretation and associate properties of the system with tests that the system will pass with probability 1 . In general, however, these properties will no longer correspond to one-dimensional subspaces of the Hilbert space, but only to higher-dimensional ones (the name 'eigenstate-eigenvalue link' becomes a bit of a misnomer in this case). ${ }^{15}$ In extreme cases, such as with two entangled electrons (where each electron's spin space is itself only two-dimensional), the individual electrons will have no non-trivial spin properties: the only test they pass with probability 1 is the trivial one testing the projection onto the whole of the Hilbert space!

\subsection{The bit commitment problem}

We shall conclude this section with an example illustrating both the notion of density operators, and some of the mystery surrounding entangled states. Because a mixed state characterises all statistical predictions of quantum mechanics for measurements on a system, it is impossible, by means of measurements performed on that system, to distinguish whether a density operator corresponds to a proper mixture or an improper mixture, or which proper mixture (if any) it corresponds to. This can be illustrated with an example from quantum information theory, the so-called bit commitment problem.

The problem is as follows: Alice commits herself to sending Bob a definite bit of information (0 or 1 ). She then sends it, and Bob receives it. How can he make sure that what she sends is, indeed, what she had committed herself

\footnotetext{
${ }^{15}$ Technically, these are all those subspaces that contain the (norm-closed) range of the density operator, i.e. the (norm-closure of) the subspace of all vectors that are images of vectors under the linear mapping defined by the density operator.
} 
to? (In whatever scheme we devise we must additionally ensure that Bob does not infer the actual bit of information any sooner than when Alice in fact sends it.) Example: Alice and Bob are on the phone, and they decide to bet on something. First Alice tosses a coin. Then Bob chooses heads or tails. Finally, Alice tells him whether it was heads or tails that had come up. The protocol is fair (or safe) if Bob is sure that Alice does not lie and if Alice is sure that Bob did not know the outcome of her toss before he chose heads or tails.

There is an obvious classical solution to this problem (assuming Bob is not an expert lock-picker): Alice writes the result of her toss on a piece of paper ( 1 for 'heads', 0 for 'tails'), puts it into a safe, sends the safe to Bob but keeps the key. After Bob has chosen heads or tails, Alice sends the key as well. The question is now whether there is a quantum solution to this problem that is rigorously fair (and could be implemented by sending just a few electrons instead of keys and safes).

Here is an attempt to realise this. (One could also phrase it in terms of polarisation states of photons, in which case Alice could send them along a more or less standard optical fibre as used in telecommunications.) Alice takes some random sequence of zeros and ones, say 1100010101110010..., and prepares a collection of electrons as follows. If the result of her coin toss (her 'bit commitment') is 'heads', she prepares the electrons one after the other as spin-up (for 1 ) and spin-down (for 0) in the $x$-direction; if her result is 'tails', she does exactly the same, but with spin states in the $y$-direction. She then sends the electrons, in sequence, to Bob.

At this point, Bob has an ensemble of electrons. We assume he knows that Alice has prepared them either in $x$-spin states or in $y$-spin states, but since the sequence is random, there are as many up states as down states on average. Since further

$$
\frac{1}{2}\left(\left|+{ }_{x}\right\rangle\left\langle+_{x}|+|-{ }_{x}\right\rangle\left\langle-{ }_{x}\right|\right)=\frac{1}{2}\left(\left|+{ }_{y}\right\rangle\left\langle+_{y}|+|-{ }_{y}\right\rangle\left\langle-{ }_{y}\right|\right),
$$

the ensemble is characterised by the maximally mixed state, irrespective of whether Alice had got heads or tails. As this characterisation gives the maximal information Bob can extract from the ensemble by making measurements, he has no way of telling whether Alice has prepared the electrons in $x$ - or $y$-spin states.

At a later stage, Alice tells Bob which way she had prepared the elec- 
trons, together with the random sequence she used. Now Bob can actually check whether Alice is telling the truth. Indeed, if he makes a sequence of measurements on the electrons, in the order they were sent, then, if the direction of his measurements is the same as the one in which they have been prepared, say $x$, he will reproduce the random sequence told him by Alice; but if the direction of his measurements is the other one, say $y$, then he will obtain a completely new random sequence which is unrelated to the first (and which Alice could thus not have anticipated). Thus, the fact that no information on top of that provided by the density operator is available, in particular about how a proper mixture has been prepared, provides a 'safe' in which the actual information about the result of Alice's toss is inaccessible without a 'key'.

But the same fact gives Alice also the possibility of cheating. Instead of sending Bob one of the two above proper mixtures, Alice can send him, say, the right-hand electrons from an ensemble of pairs prepared in the singlet state. Since it is impossible for Bob to tell whether the state he receives, namely again the maximally mixed state, is a proper or improper mixture, he sees no difference between this case and the previous case. But in this situation, Alice can wait for Bob to choose heads or tails and then perform a sequence of, respectively, spin- $x$ or spin- $y$ measurements, tell Bob she had done that before sending him the electrons (as a way of preparing the corresponding proper mixture by way of collapsing the state), and tell him the sequence of results she obtains (exchanging ones and zeros). Since results of spin measurements on pairs of electrons in the singlet state are perfectly anti-correlated, when Bob measures his electrons, he obtains, indeed, the sequence Alice has told him, not suspecting that Alice has just then collapsed the electrons into the states he measures.

Thus, the indistinguishability of proper and improper mixtures prevents Bob from finding out that Alice is cheating, while the objective difference between proper and improper mixtures (namely, in terms of the state of the composite system) makes all the difference for Alice in enabling her to cheat in the first place!

This situation turns out to be extremely general. If a protocol for bit commitment is based on the idea that a density operator could be one of two different proper mixtures, which information is then disclosed later on, then there always exists a cheating strategy based on the fact that this same density operator could be an improper mixture. This result is called the no- 
go theorem for safe bit commitment protocols (Lo and Chau 1997, Mayers 1997).

\section{Classical regime and decoherence}

The problem of the classical regime is the question of whether and how the sweeping success of classical physics (in particular on the macroscopic scale) can be explained in quantum mechanical terms. While in the philosophical literature it is the measurement problem that usually takes pride of place, the problem of the classical regime is equally important in assessing the empirical adequacy of quantum theory and its interpretations. In this section we shall look at this problem as it is generally viewed today, through the eyes of decoherence theory. To fix the ideas, however, we start with a couple of early examples of work related to this problem.

Schrödinger (1926) contributed a seminal paper on the classical regime, in which he showed that Gaussian wavepackets for the harmonic oscillator maintain their shape and size (narrow in both position and momentum) and follow the trajectories predicted by Newtonian mechanics. He believed this provided the model for the relation between 'micromechanics' and 'macromechanics'. Another early treatment of 'classical' trajectories was given by Heisenberg (1927) in his analysis of $\alpha$-particle tracks as emerging through repeated collapse of the wave function in a bubble chamber. An alternative treatment of $\alpha$-particle tracks was given by Mott (1929), who showed that the wave function of the combined system of $\alpha$-particle and gas was concentrated on configurations in which the gas was ionised along straight lines.

These examples (at least in hindsight) represent rather different approaches to understanding the problem of the classical regime, characterised by different (or potentially different) interpretational approaches. Schrödinger had an ontological commitment to the wave function. At the time, he thought of it as representing (or manifesting itself as) a charge density in 3 -dimensional space. Thus, in order to recover a classical regime, it is es-

sential in a Schrödinger-like approach to identify quantum states that are both kinematically and dynamically like classical states, i.e. for which the classical quantities such as position and momentum are both approximately 
well-defined and evolve in an approximately classical manner. ${ }^{16}$

As for Heisenberg, it appears that at the time he did not even believe in the existence of wavefunctions, but only in the transition probabilities between values of (measured) quantum mechanical observables. ${ }^{17}$ For such a view, it is essential that the transition probabilities defined by the Born rule reduce approximately to 0 or 1 for results of measurements performed along classical trajectories. Thus, such an approach (if applied consistently throughout, in particular up to the macroscopic scale) arguably aims at an instrumental recovery of the predictions of classical mechanics.

The standard interpretation and the minimal interpretation of quantum mechanics that we have introduced in section 1.2 can be seen as sanitised versions of the approaches by Schrödinger and Heisenberg, respectively. Instead, Mott's treatment is an early example of a decoherence analysis, in which no collapse need be invoked to destroy the interference between the wave components corresponding to the different trajectories. As I see it, a decoherence-based approach is best viewed as interpretationally neutral, but as providing a very powerful tool for any approach to the problem of the classical regime.

A beautiful example of the importance of the problem of the classical regime for foundational issues is given by Einstein's (1953) contribution to the Edinburgh Festschrift for Max Born. Einstein describes a macroscopic ball (of $1 \mathrm{~mm}$ diameter), bouncing elastically to and fro inside a box along the direction $x$. The wavefunction of the ball is given by a standing wave, which fills the entire box, and has a similarly spread-out distribution in momentum. According to Einstein, Born's statistical interpretation provides an adequate description of the situation for an ensemble of systems (at least according to his own reading of Born). However, an individual ball must have a welldefined macroscopic state, and that is not described by the wavefunction. To the objection that the Schrödinger equation has other solutions, that are

\footnotetext{
${ }^{16}$ It is important to add that, at least by 1927, Schrödinger was well aware that this 'charge density' was not simply a classical charge density, but a quantity that would (approximately) behave as a classical charge density only in certain respects and/or in the appropriate regime. See in particular his contribution to the 1927 Solvay conference (Schrödinger 1928), and also the discussions in Bacciagaluppi and Valentini (2009, chapter 4, esp. section 4.4) and Bacciagaluppi (2010, esp. section 4).

${ }^{17}$ See in particular Born and Heisenberg's contribution to the 1927 Solvay conference (Born and Heisenberg 1928), and the discussions in Bacciagaluppi (2008), and Bacciagaluppi and Valentini (2009, esp. chapters 3 and 6).
} 
sufficiently localised in position and momentum, Einstein replies that these solutions will spread out in time. Einstein considers also two attempts at interpretation of the wavefunction alternative to Born's. One is de BroglieBohm theory, in which a particle will have a well-defined trajectory guided by the wavefunction. ${ }^{18}$ In Einstein's example, however, the velocity of the ball will be equal to zero, so that, in Einstein's view, de Broglie-Bohm theory fails to provide the correct macroscopic description of the ball as bouncing to and fro inside the box. The other one is Schrödinger's idea of the wavefunction literally describing a wavelike nature of material particles (which in some form Schrödinger had recently returned to). In this case, however, even the macroscopic ball is a wavelike object filling the whole box, and Einstein's verdict is that also Schrödinger's reading of the wavefunction fails to do justice to the classical regime. Einstein's own conclusion is that a statistical interpretation of the wavefunction in the sense he attributes to Born is the appropriate interpretation to give to the theory.

\subsection{Coherent states and Ehrenfest's theorem}

The first question we shall discuss now is the sense in which one could talk of a quantum state as being approximately classical and behaving approximately classically, in the special case of pure quantum states. The obvious candidates are wavefunctions with a small spread both in position and in momentum (small compared to some macroscopic scale). This was Schrödinger's initial guess as to the appropriate candidates for the description of classical particles in quantum mechanics.

The Heisenberg uncertainty relations give a lower bound for the product of the spreads in position and in momentum, but for sufficiently massive ('macroscopic') systems, this in itself is a very small limitation. For instance, it is compatible with the uncertainty relations that a system have a spread in position of $10^{-13} \mathrm{~cm}$ and a spread in momentum of $10^{-13} \mathrm{~g} \mathrm{~cm} / \mathrm{s}$. If the system has a (macroscopic) mass of $1 \mathrm{~g}$, the latter corresponds to a spread in velocity of $10^{-13} \mathrm{~cm} / \mathrm{s}$. If we are merely interested in describing our system on such a macroscopic scale, we can reasonably say that the system has both a well-defined position and a well-defined momentum. Note that such a wavefunction will typically be non-zero everywhere both in position space

\footnotetext{
${ }^{18}$ Recall that Bohm (1952) had recently rediscovered and extended the pilot-wave theory by de Broglie (1928).
} 
and in momentum space. 'Small spread' means that the 'bulk' of the wavefunction is localised. Indeed, it is well-known that those wavefunctions that attain the lower bound given by the uncertainty principle are Gaussian wave packets (i.e. they have the shape of Gaussian bell curves when represented either as functions of position or as functions of momentum), and as such have infinite 'tails'. ${ }^{19}$

It is obvious, on the other hand (as in Einstein's example), that even for very massive systems there are states with macroscopically large spreads. For instance, take $\psi_{1}$ and $\psi_{2}$ to be two quantum states of a macroscopic system with very small spreads, but with macroscopically different average values of position and momentum, say $x$ and $p$ in one case, $x^{\prime}$ and $p^{\prime}$ in the other. Then the state $1 / \sqrt{2}\left(\psi_{1}+\psi_{2}\right)$ will have spreads of the order of $\left|x-x^{\prime}\right|$ and $\left|p-p^{\prime}\right|$.

An obvious question is thus whether states with small spreads in both position and momentum remain such under the quantum evolution. With regard to this, as already mentioned, Schrödinger (1926) made the following discovery. Gaussian wave functions for a harmonic oscillator (i.e. with the potential proportional to the square of position, e.g. an ideal spring) keep exactly the same shape and move exactly along the classical trajectories. These states, which are both kinematically and dynamically 'classical' are called the coherent states of the harmonic oscillator. ${ }^{20}$ Schrödinger was led by this result to think that all classical behaviour could be explained in these terms by quantum mechanics and, indeed, the result can be generalised in various ways. But we shall see that this hope was misplaced.

A simple way of generalising these results, at least in part, is as follows. For short, write $\langle A\rangle$ to mean $\langle\psi(t)|A| \psi(t)\rangle$, i.e. the average value of an operator $A$ in the state $|\psi(t)\rangle$ (see equation (5)). Then, with $m$ the mass of the particle, $Q$ and $P$ the position and momentum operators, and $V(Q)$ the operator representing the potential (which is a function of position), one

\footnotetext{
${ }^{19}$ This need not be a problem in itself, say if one interprets the wavefunction along Schrödinger's lines as manifesting itself in 3-dimensional space as a charge (or mass) density. It may become a problem if the 'tails' are themselves highly structured, as will happen in spontaneous collapse theories in the case of measurements or Schrödinger cats, as this allows for an Everettian-style criticism of the idea that such a wavefunction represents a single copy of a quasi-classical system (i.e. the tail is itself a 'tiny' live or dead cat).

${ }^{20}$ They are very important also in quantum optics, because each mode of the electromagnetic field is a harmonic oscillator.
} 
can derive the two parts of Ehrenfest's theorem:

$$
\langle P\rangle=m \frac{d}{d t}\langle Q\rangle
$$

(the average momentum is mass times the time derivative of the average position), and

$$
\frac{d}{d t}\langle P\rangle=-\langle\nabla V(Q)\rangle
$$

(the time derivative of the average momentum is equal to the average force). Thus, the average position and momentum almost obey Newton's second law, with the qualification that the classical value of the force at the average position is replaced by the average value of the force. This holds for all quantum states, but if the state has a small spread in position, the average value of the force is approximately equal to the value of the classical force. Thus, a state with a small spread in position will follow an approximately classical trajectory as long as its spread remains small (at least if the external potential in which it moves is uniform enough on the scale over which the state is spread). ${ }^{21}$

Do position spreads remain small? In the case of a Gaussian, any increase in the position spread leads to a decrease in momentum spread and vice versa. Typically, under the unitary evolution, the spread in position increases. ${ }^{22}$ In the simple case of no potentials ('free Gaussian'), if the system has macroscopic mass, the spread of the state will remain small for a very long time. For a system with mass $1 \mathrm{~g}$, starting off in a Gaussian state with position spread $10^{-13} \mathrm{~cm}$, it will take 600 years for the spread in position to increase to $10^{-4} \mathrm{~cm}$, and it will take another 6000 million million years for it to further increase to twice that size. If potentials are present, the spreading can be enhanced or counteracted, e.g. if the wave function is in a potential well it may stay trapped there. In the case of the hydrogen atom, the spreading of wave functions was pointed out to Schrödinger by Lorentz in their well-known correspondence of 1926 (published in Przibram 1963). In particular, Lorentz showed that electrons in the hydrogen atom would be spread out over their entire orbits, even for the case of high-energy orbits.

\footnotetext{
${ }^{21}$ Thus, while we might want to identify kinematically a classical state with one with small spreads in both position and momentum, it is specifically the smallness of the spread in position that determines whether this state will behave classically also in terms of its dynamics (in the sense of Ehrenfest's theorem).

${ }^{22}$ Note that this is not a strict result, but only a phenomenological arrow of time, since the Schrödinger equation is time-symmetric.
} 
The examples so far are somewhat mixed, and one might think that Schrödinger's intuition might yet prove sound at least for sufficiently macroscopic systems. That is precisely what Schrödinger replied to Einstein upon receipt of his draft for the Born Festschrift. To which Einstein replied that one could repeat the calculation taking not a $1 \mathrm{~mm}$ ball but a dust particle, and get a spread-out state within 24 hours! ${ }^{23}$

Regardless of the quantitative details, the discussion so far has presupposed that the state of our system always remain a pure state. That is, the time evolution equation of the system (the Schrödinger equation) may include external potential terms, but it includes no interaction terms. If quantum interactions are included, however, the picture changes dramatically. And that is the case we really need to discuss.

\subsection{Entanglement with the environment}

If two quantum systems do not interact, the state of each system will evolve (unitarily) within the Hilbert space that describes that system, and the state of the composite system (if initially a product state!) will always retain its product form, $|\psi(t)\rangle|\varphi(t)\rangle$. If the two systems interact, instead, the state of the composite system will evolve (unitarily) within the product Hilbert space, and in general the state of the composite system will have the entangled form

$$
\sum_{i} \alpha_{i}(t)\left|\psi_{i}\right\rangle\left|\varphi_{i}\right\rangle
$$

We can use this state in the standard way to make predictions for the composite system, as well as for either subsystem (in particular to calculate the spread in position or in momentum of either subsystem). Indeed, a measurement on a subsystem is just a special kind of measurement on the composite system, so the usual formalism applies. Equivalently, as discussed already in section 2.1, we can make predictions for measurements on a subsystem using the reduced state of that subsystem, which is an improper mixture that takes the form, say,

$$
\sum_{i}\left|\alpha_{i}\right|^{2}\left|\psi_{i}\right\rangle\left\langle\psi_{i}\right|
$$

\footnotetext{
${ }^{23}$ See Schrödinger to Einstein, no date (but between 18 and 31 January 1953), AHQP microfilm 37, section 005-012 (draft ms.) and 005-013 (carbon copy), and Einstein to Schrödinger, 31 January 1953, AHQP microfilm 37, section 005-014 (both in German).
} 
In some cases, it may be more convenient to write this as an integral, for instance over Gaussian wave packets centred at different positions (although as mentioned in section 2.1 a decomposition of the form (32) always exists). If the component states are Gaussians with macroscopically different average positions (and/or momenta), the spreads of the state now can be macroscopically large, just as with pure states that are sums of such Gaussian wave packets.

Recall that improper mixtures are not ignorance-interpretable, so that a macroscopically large spread in position or momentum that arises in this way through quantum interactions cannot be discussed away simply by applying an ignorance interpretation to the mixed state. Such a state appears to be genuinely non-classical.

Thus, we have to ask whether interactions typically lead to mixed states with large spreads, or whether we can find a regime in which these spreads remain small. Now, however, it is clearly the case that quite common interactions $d o$ in fact lead to such apparently non-classical states.

One class of interactions that lead to mixtures of macroscopically different states are measurement interactions, as with Schrödinger's (1935) own example of the cat. Although the scenario is well-known, here is the description of the thought experiment, as given by Schrödinger himself:

A cat is penned up in a steel chamber, along with the following diabolical device (which must be secured against direct interference by the cat): in a Geiger counter there is a tiny bit of radioactive substance, so small, that perhaps in the course of one hour one of the atoms decays, but also, with equal probability, perhaps none; if it happens, the counter tube discharges and through a relay releases a hammer which shatters a small flask of hydrocyanic acid. If one has left this entire system to itself for an hour, one would say that the cat still lives if meanwhile no atom has decayed. The first atomic decay would have poisoned it. The $\psi$-function of the entire system would express this by having in it the living and the dead cat (pardon the expression) mixed or smeared out in equal parts.

Such an example clearly provides a link between the problem of the classical regime and the problem of measurement. We shall postpone discussion 
of the latter, however, since the two problems are distinct. In the case of the measurement problem, we have a special case of failure or apparent failure of classicality at the kinematical level, but the dynamical behaviour of a measuring apparatus (when coupled to the measured system) is actually far from classical. The special twist of the measurement problem is that preparations and measurements are what is needed to apply quantum mechanics in the first place: if it turned out that these could not be analysed theoretically, the theory would in some sense be undermining itself. ${ }^{24}$

From the point of view of the classical regime, however, something perhaps even more startling happens, namely that very common and spontaneous interactions of a system with its environment lead to the same kind of states with large spreads.

To fix the ideas, think at first of a pair of coupled harmonic oscillators, and start them off in the non-entangled state

$$
\mid \text { ground }\rangle \mid \text { first excited }\rangle \text {. }
$$

Both classically and quantum mechanically, two coupled oscillators will recurringly exchange energy, i.e. evolve to and fro between this state and the non-entangled state $\mid$ first excited $\rangle \mid$ ground $\rangle$. But quantum mechanically, this will happen through intervening stages of the form

$$
a(t) \mid \text { ground }\rangle \mid \text { first excited }\rangle+b(t) \mid \text { first excited }\rangle \mid \text { ground }\rangle,
$$

which are entangled; and the single oscillators will be correspondingly in mixtures of their ground and first excited states. As above, these mixed states arise from quantum interactions and the ensuing entanglement. Thus, they do not allow for an ignorance interpretation.

Now imagine a harmonic oscillator coupled to a thermal bath of harmonic oscillators. It will be taking energy from and giving energy to all of them. If initially the oscillator and the bath are unentangled, the recurrence time for disentangling again could be arbitrarily long (or infinite), and in general the state of the oscillator may be a mixture of any of its energy states. Indeed, if the oscillator is assumed to be in thermal equilibrium with its environment, its quantum mechanical description is a mixture of all its energy states. The spread in position and momentum can be calculated in various ways.

\footnotetext{
${ }^{24}$ See sections $4.1-4.5$ for the theoretical discussion of measurements, and section 4.6 for the measurement problem.
} 
One rather suggestive way uses the fact that for high temperatures one can rewrite the equilibrium state as a mixture of all possible coherent states of the oscillator, with weights depending on their energy. One can thus picture the oscillator as roughly spread out over the classical trajectories corresponding to the most probable energies (see e.g. Donald 1998).

This example illustrates very well the following general idea, which I owe to Matthew Donald. While in classical statistical physics we may think of equilibrium states, at least intuitively, as describing our ignorance of the actual microstate of a system, quantum equilibrium states should generally be thought of as improper mixtures: there is no matter of fact about which pure state describes the system, and any macroscopic spreads resulting from the weighted average in the mixture are genuine non-classical features.

A macroscopic oscillator will clearly not draw in enough energy to be spread out over macroscopic scales, if the environment is, say, at room temperature (a classical oscillator will not start jittering on a macroscopic scale); but as a matter of fact, one can easily think of systems that are much more sensitive to the influence of a thermal environment, and are thus highly problematic from the point of view of justifying an approximate description in terms of classical physics. One example is a molecule of gas in equilibrium in a box. Every such molecule will be spread out over the entire volume of the box (Donald 1998). Thus, deriving classical statistical physics from quantum mechanics is part of the problem of the classical regime (cf. also Wallace 2001).

Another possible example is that of a Brownian particle suspended in a fluid. Our classical intuition is that it is tossed around by the molecules of the fluid, which influence the particle's motion in a very irregular way. If, however, the interaction of the Brownian particle with its environment is treated quantum mechanically, it would seem that its state will be an improper mixture spread over all its classically possible positions.

Radioactive decay always involves entanglement with the environment, and if the emitted radiation causes a carcinogenic mutation that kills a cat, this is only one component in a complicated entangled state (that includes not only the undecayed component, but also components describing decays at different times). The similarity with Schrödinger's cat is not accidental: this is precisely a Schrödinger cat, but arising spontaneously, without the need for the experimenter's 'diabolical device'. 
A little thought will multiply the examples. 'Environmental' interactions such as these are clearly ubiquitous. And if this is what they lead to, then it is clear that, at least in its original form, Schrödinger's approach to the problem of the classical regime is doomed to failure.

\subsection{Decoherence and the classical regime}

Luckily, the same interactions that lead to entanglement with the environment also provide at least a crucial ingredient for the resolution of the problem, because they also induce decoherence between the various classical components they superpose. ${ }^{25}$

To explain the concept of decoherence, let us first look at a very elementary example, namely the two-slit experiment. One repeatedly sends electrons or other particles through a screen with two narrow slits, the particles impinge upon a second screen, and we ask for the probability distribution of detections on the surface of the screen. In order to calculate this, one cannot just take the probabilities of passage through the slits, multiply with the probabilities of detection at the screen conditional on passage through either slit, and sum over the contributions of the two slits. There is an additional 'interference term' in the correct expression for the probability, and this term depends on both of the wave components passing through one or the other slit.

There are, however, situations in which this interference term (for detections at the screen) is not observed, i.e. in which the classical probability formula applies. This happens for instance when we perform a detection at the slits, which at least phenomenologically induces a collapse of the wavefunction. The disappearence of the interference term, however, can happen also spontaneously, when no detection at the screen is performed, for instance if sufficiently many 'stray particles' scatter off the electron between the slits and the screen. In this case, the reason why the interference term is not observed is because the electron has become entangled with the stray particles, and the results of any observation on the electron are determined by its reduced state alone. As in our discussion of reduced states in section 2.2, the probabilities for results of measurements performed only on

\footnotetext{
${ }^{25}$ This subsection is mostly based on my entry for the Stanford Encyclopedia of Philosophy (Bacciagaluppi 2003).
} 
the electron are calculated as if the wave function had collapsed to one or the other of its two components.

The intuitive picture is one in which the environment monitors the system of interest by continuously 'measuring' some quantity characterised a the set of 'preferred' states ('eigenstates of the decohering variable'). Interaction potentials are functions of position, so the preferred states will tend to be related to position, or to be in fact joint approximate eigenstates of position and momentum (since information about the time of flight is also recorded in the environment), i.e. coherent states. The localisation thus achieved can be on a very short length scale, i.e. the characteristic length above which coherence is dispersed (coherence length) can be very short. A speck of dust of radius $a=10^{-5} \mathrm{~cm}$ floating in air will have interference suppressed between (position) components with a width of $10^{-13} \mathrm{~cm}$. Even more startingly, the time scales for this process are minute. The above coherence length is reached after a microsecond of exposure to air.

One can thus argue that generically the states privileged by decoherence at the level of components of the quantum state are localised in position or both position and momentum, and therefore kinematically classical. (One should be wary of overgeneralisations, but this is certainly a feature of many concrete examples that have been investigated.)

What about classical dynamical behaviour? Interference is a dynamical process that is distinctively quantum, so, intuitively, lack of interference might be associated with classical-like dynamical behaviour. To make the intuition more precise, think of the two components of the wave going through the slits. If there is an interference term in the probability for detection at the screen, it must be the case that both components are indeed contributing to the particle manifesting itself on the screen. But if the interference term is suppressed, one can at least formally imagine that each detection at the screen is a manifestation of only one of the two components of the wave function, either the one that went through the upper slit, or the one that went through the lower slit. Thus, there is a sense in which one can recover at least one dynamical aspect of a classical description, a trajectory of sorts: from the source to either slit (with a certain probability), and from the slit to the screen (also with a certain probability). That is, one recovers a 'classical' trajectory at least in the sense that formally, the probabilities reduce to those of a classical stochastic process. 
In the case of continuous models of decoherence with interactions based on the analogy of approximate joint measurements of position and momentum, one can do even better. In this case, the trajectories at the level of the components (the trajectories of the preferred states) will approximate surprisingly well the corresponding classical (Newtonian) trajectories. Intuitively, one can explain this by noting that the preferred states are the states that themselves tend to get least entangled with the environment, so they will tend to follow the Schrödinger equation more or less undisturbed. But in fact, as we have seen from Ehrenfest's theorem, narrow wave packets follow approximately Newtonian trajectories. Thus, the resulting 'histories' will be close to Newtonian ones on the relevant scales. ${ }^{26}$

The most intuitive physical example for this are the observed trajectories of $\alpha$-particles in a bubble chamber, which are indeed extremely close to Newtonian ones, except for additional tiny 'kinks'. Indeed, one should expect slight deviations from Newtonian behaviour. These are due both to the tendency of the individual components to spread, and to the detectionlike nature of the interaction with the environment, which further enhances the collective spreading of the components (a narrowing in position corresponds to a widening in momentum). These deviations appear as noise, i.e. particles being kicked slightly off course. ${ }^{27}$ Other examples will include trajectories of a harmonic oscilator in equilibrium with a thermal bath (so the decomposition we mentioned above is not just suggestive, but in fact quite accurate), and trajectories of particles in a gas (which are a precondition for then applying classical derivations of thermodynamics from classical statistical mechanics).

Thus we see that decoherence provides us with tantalisingly classical structure, both kinematical and dynamical, at the level of components of the wave function. It is thus natural to assume that it will play a crucial role in any resolution of the problem of the classical regime. Whether it can play such a role and how, however, will depend on the interpretational approach one adopts towards quantum mechanics.

Let us take first the minimal interpretation of the theory, according to

\footnotetext{
${ }^{26}$ For a review of more rigorous arguments see e.g. Zurek (2003, pp. 28-30). Such arguments can be obtained in particular from the Wigner function formalism, as done e.g. by Zurek and Paz (1994), who apply these results to derive chaotic trajectories in quantum mechanics.

${ }^{27}$ For a very accessible discussion of $\alpha$-particle tracks roughly along these lines, see Barbour (1999, chap. 20).
} 
which quantum mechanics is about the results of preparations and measurements, and merely provides a probabilistic link between these two. If one adopts this view, the problem of the classical regime is a question about the results of measurements performed on certain 'classical', generally macroscopic systems (or possibly certain elements of their environment). Decoherence tells us that it is indeed possible to isolate a classical regime (at least one $^{28}$ ) for which appropriate measurements will reveal either actual quasi-classical trajectories, or the appearance thereof.

What we mean by this is the following: (a) if the measurements along a quasi-classical trajectory are actually carried out (as in Heisenberg's treatment of $\alpha$-particle tracks), then the results obtained will 'line up' along the quasi-classical trajectories provided by decoherence; but even if (b) the intermediate measurements are not carried out, and only the final measurement is, one can consistently assign retrospectively the whole trajectory to the system (sometimes merely guessing what the trajectories 'must have been'). This distinction is related to what is known as the movability of the Heisenberg 'cut' between observer and observed (which we discuss in the next subsection).

One will thus recover the predictions of classical mechanics, but only instrumentally. Indeed, measurements will need to be regarded as primitive even in classical mechanics, and it will be out of measurements that we will reconstruct 'objects' that 'look' and 'behave' classically (the Moon is not there if we do not look).

What of the standard interpretation? In a sense, the problem of the classical regime is more natural if one adopts this view, because if one manages to derive a classical regime within quantum mechanics in the standard interpretation, then this would recapture also the standard interpretation of classical mechanics (with measurements being derived notions). However,

\footnotetext{
${ }^{28}$ The question of uniqueness of a classical or 'quasi-classical' regime has been quite hotly debated especially in the 'decoherent histories' literature, and it appears that explicit definitions of quasi-classicality always remain too permissive to identify it uniquely. But maybe uniqueness is not strictly necessary (as nowadays often argued in the context of the Everett interpretation). For these issues see e.g. Wallace (2008).

Attempts to enforce uniqueness in other ways appear to overshoot the mark. Indeed, various 'modal' interpretations based on the biorthogonal decomposition theorem, the polar decomposition theorem, or the spectral decomposition theorem for density operators, select histories uniquely, but end up agreeing with the results of decoherence only in special cases, failing to ensure classicality in general (Donald 1998, Bacciagaluppi 2000).
} 
as we have seen, if one rejects a minimal interpretation of the formalism, but has some fuller ontological commitment to the wavefunction as describing a quantum system itself, then decoherence appears to exacerbate the problematic nature of the classical regime. Indeed, quantum interactions tend to create improper mixtures at the level of the component systems. Therefore, it would appear that they destroy classicality, as in the case of Schrödinger's cat.

As in Einstein's discussion, if one wishes to keep a fuller ontological commitment to the wavefunction, or to provide a description of individual quasi-classical systems within quantum mechanics, one will have to replace the standard interpretation (or quantum theory itself) with some alternative approach. The same broad frameworks that are usually proposed as relevant to the measurement problem appear to be useful (but note our concluding qualifications in section 5). Today's Everett interpretations are intimately connected with decoherence. Indeed, the revival of Everettian ideas can be traced back to Zeh's work on decoherence from the early 1970s, and was taken up in the philosophy literature arguably starting in the early 1990s with the work of Saunders, and later of Wallace and others. In these modern versions of Everett, either the 'many worlds' or the physical correlate of the 'many minds' are explicitly identified with the stable structures created by decoherence at the level of components of the universal wavefunction. ${ }^{29}$ Pilot-wave theories along the lines of de Broglie and Bohm also need to address explicitly the problem of the classical regime, since in general the trajectories defined in the theory are highly non-classical (see e.g. Holland 1995, chapter 6, and, for a different point of view, Allori and Zanghì 2009). At least in the non-relativistic particle theory, it would seem that the components preferred by decoherence correspond nicely with the 'full' and 'empty' waves of the theory. In Einstein's example, the macroscopic ball or dust particle will be decohered by the environment inside the box, and the system will be effectively guided by only one of the components running in opposite directions and that form the standing wave when superposed. However, it is less clear whether similar results are available in the case of quantum field theoretic generalisations (see e.g. Wallace 2008). Finally, spontaneous collapse theories might also be able to take advantage of the structures provided by decoherence (which generally operates on a much faster time-scale than spontaneous collapse), but explicit studies combining

\footnotetext{
${ }^{29}$ For a comprehensive collection of recent papers on the Everett interpretation, in particular covering the more modern developments referred to here, see Saunders et al. (2010).
} 
collapse models and decoherence are notably lacking from the literature.

\subsection{Heisenberg's 'cut'}

We conclude this section by expanding on the remarks in the last subsection on the Heisenberg 'cut'. In particular, we wish to make precise in what sense decoherence is relevant to Heisenberg's discussion of the movable cut between observer and observed (or to von Neumann's discussion of measurement chains), and in what sense it is not. This will provide also a good entry into the topic of measurement, treated in the next section.

Especially in the early 1930s, Heisenberg used to emphsise the importance of the movability of the 'cut' between the quantum and the classical domains in ensuring the consistency of quantum mechanics (cf. Heisenberg 1930, chapter 4, 1949, pp. 7-21 and 35-46, and especially 1985). Neither Heisenberg nor any of the other founding fathers of quantum mechanics believed in a rigid boundary between a quantum world, to which one could apply quantum mechanics, and a classical world, to which one could apply only classical mechanics and to which the apparatus and the observer belonged. Any parts of the world (including ostensibly 'classical' ones) could be treated quantum mechanically if one so wished. ${ }^{30}$ Consistency of the theory had to be ensured, according to Heisenberg, in the sense that applying quantum mechanics to a 'classical' part of the world should produce the same predictions as if classical mechanics had been used.

At the risk of pre-empting somewhat our discussion of measurements in the next section, let us consider a so-called 'measurement chain', e.g. the example discussed by von Neumann (1932) in his chapter on quantum measurement: we measure the temperature of a (quantum) gas using a (classical) thermometer, or we treat the interaction between the gas and the thermometer quantum mechanically, and we observe (classically) the height of the mercury column, or we treat also the interaction between the

\footnotetext{
${ }^{30}$ While this point is especially clear in Heisenberg's writings, it is clear that it was espoused also by other main exponents of what is known collectively as the Copenhagen interpretation. For instance, Bohr often applies the uncertainty relations to macroscopic pieces of apparatus in his replies to Einstein's critical thought experiments of the period 1927-1935 (Bohr 1949). And Pauli, commenting to Born on Einstein's views, is adamant that under the appropriate experimental conditions also macroscopic objects would display interference effects (Pauli to Born, 31 March 1954, reprinted in Born 1969).
} 
thermometer and the human retina quantum mechanically, and our brain registers (classically) the image on the retina, or we treat the whole physical process quantum mechanically, and it is only our consciousness that becomes ('classically') aware of the outcome (and collapses the physical state). Now, there are two senses in which we can establish the consistency of these descriptions.

First, if the successive (quantum or classical) interactions are such as to correlate perfectly the values of the temperature and the values of the quantities that are meant to record the temperature, then it follows straightforwardly that, irrespective of where the collapse postulate and Born rule are applied, one will obtain the same final results with the same probabilities. This is actually the sense in which both Heisenberg and von Neumann are interested in establishing consistency. ${ }^{31}$

Second, we can consider the influence of decoherence. Note that if no decoherence were present, then performing some other measurement on the thermometer (i.e. a measurement incompatible with that of the length of the mercury column), or somewhere further along the measurement chain, would reveal interference terms between the components of the state corresponding to different measured temperatures. The placing of the 'cut' would influence the final statistics, just as the timing of the collapse does in the case of the two-slit experiment (collapse behind the slits or at the screen). Conversely, once decoherence has kicked in at the level of the thermometer, there is no further measurement we would be able to perform in practice on the thermometer that could distinguish whether the thermometer is a classical or a quantum system. And similarly for the retina and for the brain of the observer. It is in this stronger sense that decoherence establishes that the location of the cut between the quantum and the classical domain (where the collapse postulate is applied along the measurement chain) is arbitrary. ${ }^{32}$

\footnotetext{
${ }^{31}$ Indeed, von Neumann's aim was simply to show that there always exist unitary evolutions that will produce such perfect correlations, in order to establish consistency in this first sense. Heisenberg's discussion, although technically somewhat defective (see the analysis in Bacciagaluppi and Crull 2009), is along similar lines. Note, however, that Heisenberg is particularly interested in the case of the Heisenberg microscope, where the electron interacts with a microscopic ancilla (the photon), and one considers alternative measurements on the ancilla. For Heisenberg's purposes it is thus important that interference is still present and that decoherence does not kick in until later.

${ }^{32}$ The same point is valid if we are talking about the empirical determination of when and where collapse occurs in spontaneous collapse theories. See the nice discussion in Albert (1992, chapter 5).
} 


\section{Theory and problem of measurement}

We now turn to discussing the theory and problem of measurement. We shall start by discussing measurements in some detail, using Stern-Gerlach measurements as our exemplar, and generalising the phenomenological notion of a measurement (and of a measurable quantity or 'observable') using the tools provided by the so-called POV measures. This theoretical discussion will then provide the basis for discussing the measurement problem in section 4.6.

\subsection{Discretised position measurements}

Quite surprisingly, there is no perfect analogue for the collapse postulate in the case of measurements of continuous quantities, such as position. Naively one would expect a wavefunction $\psi(x)$ to collapse to a (renormalised) Dirac $\delta$-function centred at some point $q$, i.e. to $\psi(x) \delta(x-q)$, with a Born probability density given by $|\psi(q)|^{2}$. The problem with this is the mathematical fact that any function that is non-zero at a single point has square integral 0 , and is thus identified with the zero vector. Dirac's famous $\delta$-functions are thus not actually quantum states, so, trivially, one cannot collapse a state to a $\delta$-function.

This was recognised already by von Neumann (1932), who used discretisation procedures to describe measurements of position. For instance, we can (ideally) test for whether a wave function lies in the subspace of all squareintegrable functions that are non-zero in the interval $\left[x_{1}, x_{2}\right]$. If the test is positive, the original wave function $\psi(x)$ will collapse to $\chi_{\left[x_{1}, x_{2}\right]}(x) \psi(x)$ (suitably renormalised), ${ }^{33}$ with probability

$$
\int \chi_{\left[x_{1}, x_{2}\right]}(x) \psi(x) d x
$$

(This suggestion is so obvious that the problematic nature of the collapse postulate for continuous quantities often goes unnoticed.)

Such discretised measurements of position are all we need to analyse explicitly how a spin measurement works, and in fact to generalise it to

\footnotetext{
${ }^{33}$ The function $\chi_{\left[x_{1}, x_{2}\right]}(\mathbf{x})$ is the so-called characteristic function of the interval $\left[x_{1}, x_{2}\right]$, i.e. the function that is 1 on the interval and 0 outside.
} 
include more realistic kinds of spin 'measurements'.

\subsection{Ideal spin measurements}

Let us first describe the case of an ideal measurement of spin. Note that a system with both spin and position degrees of freedom is described using the tensor product of the Hilbert spaces used to describe a 'pure' spin- $1 / 2$ system (a two-dimensional complex Hilbert space) and a spinless particle (the Hilbert space of Schrödinger's wavefunctions), just as if one were composing two separate systems.

Take an electron that we assume to be initially in a state

$$
|\varphi\rangle \otimes \psi
$$

What this means is that the electron is described as having a spin state, given by the vector $|\varphi\rangle$ in the two-dimensional spin space of the electron, as well as a wavefunction, $\psi$.

Now suppose we want to perform a measurement of spin in some given direction, and that with respect to this spin basis, $|\varphi\rangle=\alpha|+\rangle+\beta|-\rangle$, so that (36) equals

$$
\alpha|+\rangle \otimes \psi+\beta|-\rangle \otimes \psi
$$

If we pass the electron through an ideal Stern-Gerlach magnet, the evolution of the state will be described by the appropriate Schrödinger equation, which is unitary. Therefore, we can consider separately the deflection of the two components and superpose the results. We obtain

$$
\alpha|+\rangle \otimes \psi_{+}+\beta|-\rangle \otimes \psi_{-}
$$

(where $\psi_{+}$and $\psi_{-}$are suitably deflected versions of $\psi$ ). We see that the spin degree of freedom of the electron is now entangled with its position degrees of freedom.

We now detect the electron on a screen, i.e. perform a position measurement. Indeed, we perform a discretised measurement of position, because we only need to distinguish whether the electron hits the half of the screen associated with the up or down component of the spin (which, as mentioned in the footnote on p. 4, depending on the experimental setup might or might 
not coincide with the upper or lower half of the screen, respectively). Assuming that $\psi_{+}$and $\psi_{-}$do not overlap, the standard collapse postulate and Born rule, applied to the detection of the electron on the screen, will yield either

$$
|+\rangle \otimes \psi_{+} \quad \text { with probability }|\alpha|^{2}
$$

or

$$
|-\rangle \otimes \psi_{-} \quad \text { with probability }|\beta|^{2},
$$

and thus we can actually derive the collapse postulate and Born rule for the spin measurement from the collapse postulate and Born rule for the discretised measurement of position.

\section{3 'Unsharp' spin measurements}

Real experiments, however, will not yield exactly the above result. Let us return to the discussion of our Stern-Gerlach example. It is a fact that wavefunctions, even if at any one time they can be zero outside of a given interval, will (typically) spread instantaneously out to infinity, so that while we could expect the bulk of $\psi_{+}$and $\psi_{-}$to be concentrated each on one half of the screen, they will have 'tails' spreading out to the 'wrong' half of the screen, say

$$
\psi_{+}=\psi_{++}+\psi_{+-} \quad \text { and } \quad \psi_{-}=\psi_{--}+\psi_{-+} .
$$

Here, $\psi_{++}$is meant to represent that part of $\psi_{+}$that is distributed over the half-screen associated with the up result, and $\psi_{+-}$the part that is distributed over the half-screen associated with the down result; and similarly for $\psi_{--}$and $\psi_{-+}$. We shall assume for simplicity that

$$
\int\left|\psi_{+-}\right|^{2}=\int\left|\psi_{-+}\right|^{2}=: \varepsilon
$$

and thus also

$$
\int\left|\psi_{++}\right|^{2}=\int\left|\psi_{--}\right|^{2}=1-\varepsilon
$$

In this case, applying the collapse postulate and Born rule to detecting the electron on the screen yields either

$$
\alpha|+\rangle \otimes \psi_{++}+\beta|-\rangle \otimes \psi_{-+}
$$


or

$$
\alpha|+\rangle \otimes \psi_{+-}+\beta|-\rangle \otimes \psi_{--}
$$

(both to be suitably renormalised), with probabilities

$$
|\alpha|^{2}(1-\varepsilon)+|\beta|^{2} \varepsilon
$$

and

$$
|\alpha|^{2} \varepsilon+|\beta|^{2}(1-\varepsilon)
$$

respectively.

We see that the effect of the measurement on the spin state of the electron is no longer simply given by the standard collapse postulate. Indeed, the two possible states of the electron after the measurement are not even product states, so that the spin of the electron is still entangled with its spatial degrees of freedom, and the spin part of the electron is collapsed to an improper mixture: either

$$
|\alpha|^{2}(1-\varepsilon)|+\rangle\left\langle+\left.|+| \beta\right|^{2} \varepsilon \mid-\right\rangle\langle-|
$$

or

$$
|\alpha|^{2} \varepsilon|+\rangle\left\langle+\left.|+| \beta\right|^{2}(1-\varepsilon) \mid-\right\rangle\langle-|
$$

with the same probabilities (46) and (47) (also here, we need to suitably renormalise, since the weights in each decomposition need to sum to 1).

Note that these are the states we obtain if we, indeed, know the result of the spin measurement, and can select one of these two final states on the basis of the measurement result (thus performing a so-called selective measurement). If we do not know the outcome of the spin measurement, then future predictions for spin measurements on the electron will use a state that is itself a (proper) mixture of the two corresponding density operators. We can obtain this by simply adding the two (unnormalised) states (48) and (49), to yield

$$
|\alpha|^{2}|+\rangle\left\langle+\left.|+| \beta\right|^{2} \mid-\right\rangle\langle-|
$$

This is now a case of non-selective measurement, in which we obtain a mixed state that is partially ignorance-interpretable. But - as in the case of the bit commitment problem of section 2.3 - we need to know the past history of the system (how the state has been prepared), in order to know how and how far to interpret this mixed state in terms of ignorance. If the measurement is ideal, then the correct decomposition of the state is in terms of spin-up 
or spin-down; if the measurement is correctly modelled by the above, the correct decomposition is given in terms of (48) and (49).

In this example (where we have combined an ideal Stern-Gerlach magnet with a more realistic position state), we see that the probabilities in (50) are independent of the shape of the position state (and indeed, of whether it is 'ideal' or 'realistic'). One easily realises that even more general transformations on the spin state of the electron can be induced by a detection on the screen, if one considers that the Stern-Gerlach magnetic field itself is not 'ideal' (in order to satisfy the Maxwell equations, it cannot be perfectly homogeneous in the directions perpendicular to that of measurement). Or, indeed, if one considers that one could have chosen, at least in principle, any other unitary coupling between the spin and position degrees of freedom of the electron before proceeding to the detection on the screen.

\subsection{General phenomenology of measurements}

The above examples of various kinds of spin measurements serve as perfect illustrations of the general phenomenology and theory of measurements in quantum mechanics. As discussed in section 1.1, measurements are phenomenologically captured by the collapse postulate, which describes transformations on the state of the measured system, and the Born rule, which gives the probabilities for such transformations. Both rules need to be generalised. We shall sketch this generalisation here, but only in the discrete, finite-dimensional case.

Let us first slightly redescribe the collapse postulate and Born's rule for the case of the standard measurements of section 1.1. Take a family of mutually compatible quantum mechanical tests, corresponding to a family of mutually orthogonal subspaces of the Hilbert space. (If they do not span already the whole Hilbert space, we can add to the family the orthogonal complement of their span, corresponding to the system testing negatively to all the tests.) The corresponding projection operators form a so-called (PV, or projection-valued) resolution of the identity:

$$
\sum_{i} P_{i}=\mathbf{1}
$$

where $\mathbf{1}$ is the identity operator on the Hilbert space. In this case, we also 
talk of a PV-observable. ${ }^{34}$

In the case of a selective measurement of this PV-observable, with outcome $i$, the state of the system collapses as:

$$
|\psi\rangle \mapsto P_{i}|\psi\rangle,
$$

or more generally, writing $\rho$ for the initial state to cover also the case when it might not be pure:

$$
\rho \mapsto P_{i} \rho P_{i}
$$

(in both cases with suitable renormalisation). The probabilities for the collapses (52) and (53) are, respectively, $\left\langle\psi\left|P_{i}\right| \psi\right\rangle$ and

$$
\operatorname{Tr}\left(P_{i} \rho P_{i}\right)=\operatorname{Tr}\left(\rho P_{i}\right) .
$$

(Note that the trace is cyclic, i.e. $\operatorname{Tr}(A B)=\operatorname{Tr}(B A)$ for any two operators, and that $P_{i}^{2}=P_{i}$ for projections.)

In the case of a non-selective measurement, the collapse takes the form

$$
\rho \mapsto \sum_{i} P_{i} \rho P_{i}
$$

(already normalised, because of (51)).

In the case of the more realistic spin measurements just discussed, instead of the transformation (52), we have a transformation to an (improper) mixture, which we can write as

$$
|\psi\rangle\left\langle\psi\left|\mapsto \sqrt{1-\varepsilon} P_{+}\right| \psi\right\rangle\left\langle\psi\left|\sqrt{1-\varepsilon} P_{+}+\sqrt{\varepsilon} P_{-}\right| \psi\right\rangle\langle\psi| \sqrt{\varepsilon} P_{-}
$$

or

$$
|\psi\rangle\left\langle\psi\left|\mapsto \sqrt{\varepsilon} P_{+}\right| \psi\right\rangle\left\langle\psi\left|\sqrt{\varepsilon} P_{+}+\sqrt{1-\varepsilon} P_{-}\right| \psi\right\rangle\langle\psi| \sqrt{1-\varepsilon} P_{-},
$$

depending on the outcome, with probabilities given by the trace of (56) or of $(57)$, respectively.

\footnotetext{
${ }^{34}$ Instead of talking of resolutions of the identity, one can also talk of PV 'measures', in the sense that (analogously to a probability measure), one can assign to each 'event' (subset $I$ of the indices labelling the results) a corresponding projection $\sum_{i \in I} P_{i}$. One will talk similarly of POV measures when the requirement that the elements of the resolution of the identity be projections is relaxed.
} 
In the most general case, the transformation (52) or (53) takes the form of a so-called operation, or completely positive map:

$$
\rho \mapsto \sum_{j} A_{i_{j}} \rho A_{i_{j}}^{*}
$$

with suitable operators $A_{i_{j}}$ for each outcome $i$. (If there is only one $A_{i_{j}}$ corresponding to the outcome $i$, the operation is said to be 'pure', because it maps pure states to pure states.)

The corresponding probabilities are given by

$$
\operatorname{Tr}\left(\sum_{j} A_{i_{j}} \rho A_{i_{j}}^{*}\right)=\operatorname{Tr}\left(\rho \sum_{j} A_{i_{j}}^{*} A_{i_{j}}\right)=\operatorname{Tr}\left(\rho E_{i}\right)
$$

where we have defined the so-called effect $^{35} E_{i}$ as

$$
E_{i}:=\sum_{j} A_{i_{j}}^{*} A_{i_{j}}
$$

In the non-selective case, the transformation (55) becomes

$$
\rho \mapsto \sum_{i} \sum_{j} A_{i_{j}} \rho A_{i_{j}}^{*}
$$

And the normalisation of the probabilities (59) yields the analogue of (51), namely

$$
\sum_{i} E_{i}=\mathbf{1}
$$

i.e. the $E_{i}$ form an effect-valued (or POV, or positive-operator-valued) resolution of the identity (or POV-observable).

Interesting special cases are obtained when, as in the case of the spin measurements above, the operations are combinations of the projections from a PV-observable (so-called 'unsharp' measurements of the corresponding PV-observable); or when the effects $E_{i}$ are in fact mutually orthogonal projections, but the corresponding operations are not simple projections, but have a more general form ('disturbing' measurement of the corresponding PV-observable). Other cases of POV-observables can be interpreted as

\footnotetext{
${ }^{35}$ Technically, an effect is a positive operator with spectrum contained in the interval $[0,1]$.
} 
corresponding to sequences of measurements of PV-observables (or of other POV-observables), yet others as corresponding (in certain specific senses) to joint unsharp measurements of incompatible PV-observables. The closer relation between a measurement and a single self-adjoint operator mentioned in section 1.1 is clearly lost in the general case.

These transformations provide the general form of the phenomenological collapse postulate, and the corresponding probabilities the general form of the phenomenological Born rule. The above discussion of spin measurements, however, illustrates also the general theoretical description of such measurements. Indeed, one can show (this is know as the Naimark dilation theorem) that any completely positive map on the states of the measured system can always be obtained by suitable interaction with some other system, followed by a PV-measurement on this other system (i.e. a transformation of the form (53) or (55), where it should again be emphasised that the $P_{i}$ need not be one-dimensional projections). This other system can be thought of either as a generally microscopic ancillary system or degree of freedom (e.g. the position of the electron in a Stern-Gerlach measurement, or the photon in the Heisenberg microscope), or as an 'indicator variable' or 'pointer variable' of a generally macroscopic measuring device. We shall see this in detail (for the case of ideal measurements) in subsection 4.5.

POV-observables provide a very powerful tool for describing the phenomenology of quantum mechanical measurements. And they have become a completely standard tool in various branches of quantum physics (e.g. quantum information theory).

For instance, it is well-known that using a measurement of a single PVobservable it is impossible to reconstruct completely the quantum state describing an ensemble of systems. (If the PV-observable is spin in some direction, and if the state is pure, say $\alpha|+\rangle+\beta|-\rangle$ in that basis, the measurement statistics will determine only the absolute values of the coefficients $\alpha$ and $\beta$, not their relative phases.) But there are single POV-observables (so-called informationally complete observables) that allow such a reconstruction. A simple example is given by the resolution of the identity

$$
\frac{1}{3} P_{+}^{x}+\frac{1}{3} P_{-}^{x}+\frac{1}{3} P_{+}^{y}+\frac{1}{3} P_{-}^{y}+\frac{1}{3} P_{+}^{z}+\frac{1}{3} P_{-}^{z},
$$

which intuitively pools together the information provided by measurements of spin in the three directions $x, y$ and $z$ (and can be seen as one sense of a joint unsharp measurement of the three PV-observables (Cattaneo et 
al. 1997)). Indeed, such a POV-measurement can be performed simply by throwing a die and measuring spin in the direction $x, y$ or $z$ depending on whether the die shows up 1,2 or $3(\bmod 3)$. Note, however, that from the Naimark dilation theorem we also know that there is a single interaction with an ancilla or measuring device that will implement on the electron any set of six operations needed to measure the POV-observable (63).

Note that Gleason's theorem can be formulated also in terms of probability measures over the outcomes of all possible POV experiments, yielding again the quantum mechanical mixed states as the most general states defining probabilities for the outcomes of such experiments. ${ }^{36}$

We conclude by mentioning one example of continuous POV measurements, the so-called 'unsharp' measurements of position, which provide a continuous alternative to von Neumann's discretisation procedures. The wavefunction $\psi(x)$ collapses upon measurement to a wavefunction

$$
\alpha(x-q) \psi(x)
$$

(suitably renormalised), where $\alpha(x-q)$ is not a $\delta$-function but a normalised Gaussian centred at $q$. The probability density for the collapse is given by

$$
\int|\alpha(x-q) \psi(x)|^{2} d x
$$

This POV-measurement has the intuitive properties of a measurement of position, in that after the collapse the wavefunction is concentrated around the point $q$. Readers may recognise this as the family of operations that take place spontaneously in the spontaneous collapse theory by Ghirardi, Rimini and Weber (1986). ${ }^{37}$

\subsection{The standard model of measurement}

The model of measurement that underlies standard discussions of the measurement problem (although usually phrased mainly in terms of ideal measurements) is directly related to the theoretical description based on the dilation theorem, as follows.

\footnotetext{
${ }^{36}$ In this formulation, the theorem holds in all dimensions (Busch 2003).

${ }^{37}$ For further details of POV observables, we refer the reader to standard references, e.g. Busch, Grabowski and Lahti (1995).
} 
In the ideal case, one takes the a basis of eigenvectors of the observable one wishes to measure on the system of interest, $\left\{\left|\varphi_{i}\right\rangle\right\}$, and couples it oneto-one to an orthonormal family of states $\left\{\left|\psi_{j}\right\rangle\right\}$ of the apparatus, in the sense that for some 'ready state' $\left|\psi_{0}\right\rangle$ of the apparatus,

$$
\left|\varphi_{i}\right\rangle \otimes\left|\psi_{0}\right\rangle \mapsto\left|\varphi_{i}\right\rangle \otimes\left|\psi_{i}\right\rangle
$$

for all $i$. This is indeed possible through a single unitary evolution, because it is simply a requirement that orthonormal states be mapped into orthonormal states.

The outcomes of the measurement are assumed to correspond to orthogonal subspaces (not necessarily one-dimensional), or their corresponding projections $P_{k}$, each containing one or more of the $\left|\psi_{i}\right\rangle$ (depending on the 'resolution' of the measurement). If each outcome corresponds to a single $\left|\varphi_{i}\right\rangle$, the measurement is said to be maximal. ${ }^{38}$

Under this coupling, an arbitrary state of the system will interact with the apparatus in the ready state as

$$
\sum_{i} \alpha_{i}\left|\varphi_{i}\right\rangle \otimes\left|\psi_{0}\right\rangle \mapsto \sum_{i} \alpha_{i}\left|\varphi_{i}\right\rangle \otimes\left|\psi_{i}\right\rangle
$$

If the measurement is maximal, applying the standard collapse postulate to the pointer observable will now yield any one of the states

$$
\left|\varphi_{i}\right\rangle \otimes\left|\psi_{i}\right\rangle
$$

with probability $\left|\alpha_{i}\right|^{2}$. If the measurement is non-maximal, more than one $\left|\psi_{i}\right\rangle$ will lie in the subspace associated with the measurement outcome, and the collapse will yield some superposition of the states (68). ${ }^{39}$

More generally, a measurement will involve an arbitrary coupling between the system of interest and the apparatus, so that the final state of the

\footnotetext{
${ }^{38}$ Note that the corresponding subspace in the apparatus Hilbert space need not be one-dimensional: in the case of the spin measurements of section 4.2 , we had infinitedimensional projections onto the upper or lower half of the detection screen. Given that the 'apparatus' will usually be a macroscopic system, the idea that a reading should correspond to a large subspace of its state space rather than to a single state is quite appealing. A reading ought to correspond rather to a macroscopic state of the apparatus than to a microscopic state, and a macroscopic state could well be represented by an appropriate subspace $P_{k}$.

${ }^{39}$ Note that in this case the system is collapsed to an improper mixture of the states $\left|\varphi_{i}\right\rangle$.
} 
composite will have the form

$$
\sum_{i} \sum_{j} \alpha_{i j}\left|\varphi_{i}\right\rangle \otimes\left|\psi_{j}\right\rangle
$$

or performing the sum over $i$ first,

$$
\sum_{j}\left(\sum_{i} \alpha_{i j}\left|\varphi_{i}\right\rangle\right) \otimes\left|\psi_{j}\right\rangle
$$

Defining $\beta_{j}$ as the norm of $\sum_{i} \alpha_{i j}\left|\varphi_{i}\right\rangle$, and $\left|\varphi_{j}^{*}\right\rangle$ as $\frac{1}{\beta_{j}} \sum_{i} \alpha_{i j}\left|\varphi_{i}\right\rangle$, (70) can be rewritten as

$$
\sum_{j} \beta_{j}\left|\varphi_{j}^{*}\right\rangle \otimes\left|\psi_{j}\right\rangle
$$

Applying the standard collapse postulate to the pointer observable will now yield any one of the states

$$
\left|\varphi_{j}^{*}\right\rangle \otimes\left|\psi_{j}\right\rangle
$$

with the corresponding probability $\beta_{j}^{2}$ (or some superposition thereof if more than one $\left|\psi_{j}\right\rangle$ lies in the subspace associated with the measurement outcome).

\subsection{The measurement problem}

In this section, we shall build on the theory of measurement we have just sketched, and describe the measurement problem of quantum mechanics. The phrase 'measurement problem' denotes a complex of interrelated questions, but we shall take the following to be its core: whether the practical rules of quantum mechanics (collapse postulate and Born rule) are derivable from first principles, by applying the theory (in particular the dynamics of the theory, as given by the deterministic Schrödinger equation) to a measurement situation, i.e. a situation in which we have an appropriate interaction between a system and a measuring apparatus.

As we have seen generalising the example of the Stern-Gerlach measurement, a theoretical description of a measurement can indeed be given by coupling the system of interest (the spin of the electron) to some 'indicator' variable (the position of the electron on the upper or lower half of the screen). And we have also seen that the collapse postulate and Born rule for the system (in their most general form) can be obtained by applying 
the collapse and Born rule (in their more restricted form) to the indicator variable itself.

Suppose that from an appropriate application of the Schrödinger equation, and without explicitly invoking the collapse postulate and the Born rule for the indicator variable, one could derive that in the correct fraction of cases the final state after a measurement is given by (68) or (72), rather than by (67) or (71). Then the collapse and the Born rule would be derivable from first principles, irrespective of whether one adopts the minimal or standard interpretation of the theory.

Indeed, under the standard interpretation, states such as (68) or (72) - or even appropriate superpositions of such states - correspond to the apparatus possessing an intrinsic property indicating a definite outcome (a subspace $P_{k}$ representing an appropriate macrostate of the apparatus). And under the minimal interpretation, these same states mean that the apparatus has a sure-fire dispositional property to be seen as indicating a definite outcome if somebody looks.

However, this is not true if the the description just given in section 4.5 is correct. Just like in the bit commitment problem of section 2.3, where there is an objective difference between the case in which Alice sends a statistical mixture of electrons in various spin states, and the case in which she sends electrons from entangled pairs (a difference enabling her to cheat), so in the case of the standard model of measurement there is an objective difference between the case of a statistical mixture of states associated with different measurement results and states in which the macroscopic outcome is entangled with the microscopic value of the measured observable. The theoretical description of measurement in terms of a unitary interaction has merely shifted the place of application of the phenomenological rules. Thus, if it is a correct description of the process of measurement, it does not provide a derivation of the collapse postulate and Born rule, whether the interpretation of choice is the minimal interpretation or the standard interpretation.

Before discussing this further, we should pause to consider whether we have been misled by the power of the dilation theorem and been overly rash in adopting this model of measurement. That is, we should see whether the negative result just described is merely an artefact of the model of measurement adopted. 
What could count as a derivation of the collapse postulate and Born rule from the Schrödinger equation? At first, it might seem conceptually mistaken even to pose such a question. How can a probabilistic process ever be derived from a deterministic one? According to von Neumann, the differences run even deeper, in that the former is a thermodynamically irreversible process, while the latter is reversible. ${ }^{40}$ On the other hand, in the case of classical thermodynamics and statistical mechanics, we are familiar with the claim that a phenomenologically irreversible theory can be reduced (in some appropriate sense) to an underlying reversible one. The obvious first attempt at answering the problem is thus to point out that it is perfectly possible for a deterministic evolution to underpin statistical results, if we consider statistical states (that is, genuinely statistical states, which are proper mixtures) rather than pure states. That is, while the Schrödinger evolution maps pure states into pure states, it is perfectly possible to obtain a final state that is a proper mixture of different readings of the apparatus, if the initial state is not pure but itself a proper mixture.

The intuition here is that the initial state of system and apparatus should be given not by a product of pure states, but more realistically by a state of the form $\left|\psi_{0}\right\rangle \otimes \rho_{0}$, where $\rho_{0}$ is a suitable statistical state of the apparatus. Indeed, any realistic apparatus will arguably be a macroscopic object, and thus its exact microstate will not be specifiable. Instead, the state of the apparatus will be given only in terms of certain macroscopic parameters, analogously to the macrostates of statistical mechanics, and thus for instance to be described as lying in some subspace $P_{0}$ or as some appropriate proper mixture of microstates. ${ }^{41}$

For simplicity, let us stick to our Stern-Gerlach example, even though the 'indicator' variable (the position of the electron) is not itself macroscopic. ${ }^{42}$ Imagine that initially we are not able to prepare the wave function $\psi$ for the spatial degrees of freedom of the electron, but only a proper mixture $\rho=\int_{\Lambda} \beta_{\lambda} \rho_{\lambda} d \lambda$, where each $\rho_{\lambda}$ corresponds itself to a wavefunction $\psi_{\lambda}$ (a pure state) localised within the spread of the original $\psi$. Imagine further

\footnotetext{
${ }^{40}$ Von Neumann's characterisation is based on an extensive thermodynamic analysis, which we shall not enter into, but it should be immediately clear that the transformation (61) is not time-reversible.

${ }^{41}$ We shall assume this for the sake of argument, even though we have suggested in section 3.2 that these mixtures might be improper in the first place.

${ }^{42}$ Recall that on the minimal interpretation the indicator variable is merely a variable that if measured will produce the result that the apparatus reads either up or down. The position of the electron fulfills this role perfectly.
} 
that $\rho$ decomposes into

$$
\rho=\int_{\Lambda_{+}} \mu_{\lambda} \rho_{\lambda} d \lambda+\int_{\Lambda_{-}} \mu_{\lambda} \rho_{\lambda} d \lambda
$$

where the weights $\mu_{\lambda}$ are related to the coefficients in the decomposition (37) as

$$
\int_{\Lambda_{+}} \mu_{\lambda} d \lambda=|\alpha|^{2} \quad \text { and } \quad \int_{\Lambda_{-}} \mu_{\lambda}=|\beta|^{2} .
$$

Here $\Lambda_{+}$is the set of indices for which $|\psi\rangle \otimes \rho_{\lambda}$ evolves to some final state entirely contained in the subspace corresponding to $\mathbf{1} \otimes P_{+}$(i.e. the projection onto the half of the screen associated with 'up'). And correspondingly for $\Lambda_{-}$.

Since the initial mixture was by assumption ignorance-interpretable, also the final state is a proper mixture such that in a fraction $|\alpha|^{2}$ of cases, the electron has ended up on the half of the screen associated with 'up', and in a fraction $|\beta|^{2}$ of cases, the electron has ended up on half of the screen associated with 'down', as desired.

The problem with this obvious strategy is that it does not work in general. Indeed, if the initial state of the electron is not (37) but, say,

$$
\left|\varphi^{\prime}\right\rangle=\gamma|+\rangle+\delta|-\rangle
$$

then, even assuming that each $\rho_{\lambda}$ still ends up on one or the other half of the screen, it is not clear why the new sets $\Lambda_{+}^{\prime}$ and $\Lambda_{-}^{\prime}$ into which the set $\Lambda$ splits should again satisfy (74) with $\gamma$ and $\delta$ substituted for $\alpha$ and $\beta$. Indeed, this constraint is impossible to satisfy for all initial spin states $|\varphi\rangle$ if the temporal evolution is unitary. ${ }^{43}$ The apparatus would have to conspire to know in advance what spin state it is meant to measure in order to be in the appropriate statistical mixture of microstates that will produce the desired outcomes with the desired frequencies. ${ }^{44}$

\footnotetext{
${ }^{43}$ Note also that even if this were possible, such a solution to the measurement problem would run into trouble when trying to reproduce the experimental violations of the Bell inequalities, at least unless the microstates of the apparatuses are correlated before the measurements.

${ }^{44}$ Arguably, the only loophole is if one considers models in which the initial correlations between the microstate of the apparatus and the state of the system (and between the microstates of different apparatuses) are explained in retrocausal terms, and thus are no longer conspiratorial. The models of measurement by Schulman (1997) are probably best understood in this way. For more general discussion of retrocausal models in quantum mechanics, see Price (1996).
} 
For the case of ideal measurements, the fact that the measurement problem cannot be solved by invoking an initial mixed state of the apparatus was already discussed by von Neumann (1932, section VI.3), who remarks that this idea was often proposed as a solution to the measurement problem. ${ }^{45}$ (It is periodically 'rediscovered', which only shows that von Neumann's book is often referred to but still not widely read.) Von Neumann used this to support his claim that one needs indeed two different kinds of processes (namely collapse and unitary evolution) to describe the behaviour of quantum systems with and without measurements. This 'insolubility theorem', as it is now known, has since been widely generalised, in particular to include also measurements of POV observables. ${ }^{46}$

Thus we are left with our original conclusion, irrespective of the model of measurement we choose. In a nutshell, measurements understood as quantum interactions magnify quantum superpositions to the macroscopic level (because of the linearity of the dynamics), and thus do not lead to the phenomenologically correct behaviour (collapse postulate and Born rule). If we apply the Schrödinger equation to describe the measurement process, then we do not obtain states that would seem to include definite measurement results, but superpositions of such states, nor do we obtain any kind of probabilistic distributions over final states.

Under the minimal interpretation, this might not be very satisfactory, but need not be particularly troubling, since the interpretation only seeks to provide an instrumentalist reading of the theory. And in various variants of the Copenhagen interpretation, one can argue that one should in fact expect measurements - or the quantum-classical interface - to display a peculiar behaviour. What is essential, on these interpretations, is consistency between different choices of when and where to apply the collapse postulate and the Born rule. And, as we discussed in section 3.3, this consistency is ensured by decoherence, or in a weaker sense by the existence of perfect correlations along a measurement chain. ${ }^{47}$

\footnotetext{
${ }^{45}$ Historical puzzle: who is von Neumann referring to? Someone like Schrödinger who suggested matter should be literally described by wavefunctions? Or something like the early Copenhagen 'disturbance' theory of measurement?

${ }^{46}$ See e.g. Fine (1970), Brown (1986), Busch and Shimony (1996) and Bassi and Ghirardi (2000).

${ }^{47}$ I would suggest, however, that if one considers the in-principle possibility of performing arbitrary measurements unimpeded by decoherence, then problems of consistency arise again in the context of thought experiments of the type of Wigner's friend.
} 
As a matter of fact, von Neumann considered the measurement problem to be purely the question of whether such consistency (in the weaker sense) could be ensured, and his treatment of the measurement problem consists precisely in showing that unitary evolutions exist that will produce the perfect correlations (i.e., essentially, in showing that the standard model of measurement is well-defined). Collapse could occur when the thermometer records the temperature of the gas, or when the length of the mercury column is recorded in the photons travelling to the eye, or in our retina, or along the optic nerve, or when ultimately consciousness is involved. If all of these possibilities are equivalent as far as the final predictions are concerned, von Neumann can maintain that collapse is related to consciousness while in practice applying the collapse postulate at a much earlier (and more practical) stage in the description.

From the point of view of the standard interpretation, however, the problem is serious, because in the state (67) or (71) the system and the apparatus are entangled, and the mixed state resulting for the apparatus is not ignorance-interpretable. Thus, the apparatus does not have a reading under the standard interpretation. As we have also seen, in the case of the standard interpretation decoherence does not help; if anything it makes the situation even worse, because it will produce such macroscopic improper mixtures even independently of observer-engineered measurement situations.

Insofar as the standard interpretation is meant as an approach to quantum mechanics that treats it as a fundamental theory, rather than as a phenomenological theory, we see that the standard interpretation fails. In particular, it fails to support a theoretical analysis of the process of measurement that ensures that measurements have definite outcomes, let alone one that enables one to rederive the phenomenological rules for the description of measurements (the collapse postulate and the Born rule). Everett theories, pilot-wave theories and spontaneous collapse theories are again the options of choice if one wishes to provide a solution to the measurement problem rather than a minimalist or (neo)-Copenhagen dissolution, ${ }^{48}$ but a detailed discussion of these goes beyond the scope of this article.

\footnotetext{
${ }^{48}$ As a prominent example of a neo-Copehagen view, one can take the 'quantum Bayesianism' of Fuchs and co-workers (e.g. Fuchs 2010).
} 


\section{Conclusion}

We have discussed two of the main interpretational problems of quantum mechanics, both engendered by the nature of quantum mechanical entanglement, and the consequent failure of the ignorance interpretation of reduced states.

The two problems are equally important if one wishes to give a foundationally adequate reading of quantum mechanics. We are not here in the business of discussing what a foundationally adequate reading of quantum mechanics might be. The minimal interpretation, while not being entirely satisfactory, will arguably count as adequate if one has an instrumentalist picture of science. More sophisticated Copenhagen or neo-Copenhagen views may also find it easier to negotiate these two problems. ${ }^{49}$ Yet more robust ontological requirements will prompt one to seek a more successful replacement for the standard interpretation of quantum mechanics, with the help of decoherence and usually along the lines of de Broglie-Bohm, collapse or Everett.

A solution to the problem of the classical regime, however, will not automatically be also a solution to the measurement problem (and vice versa). While pieces of apparatus are generally macroscopic systems or arguably at least kinematically classical systems, their dynamical behaviour in probing the quantum world is decidedly non-classical, and solving the dynamical aspects of the measurement problem is thus distinct from deriving approximately Newtonian trajectories. For instance, modern-day Everettians can use the results of decoherence in an extremely effective way, both towards the solution of the problem of the classical regime and towards that of the measurement problem. But the Everettian solution to the measurement problem relies more heavily on a successful derivation of the Born rule (e.g. the decision-theoretic approach proposed by Deutsch (1999) and Wallace (2007)). Should the critics of the Deutsch-Wallace approach prove correct (e.g. Lewis 2010), Everettians might still be lacking a derivation of the Born rule from first principles, and thus a full solution to the measurement problem.

Conversely, a solution to the measurement problem will not automatically be a solution to the problem of the classical regime. For instance,

\footnotetext{
${ }^{49}$ Modulo the caveat about Wigner's friend in section 4.6 .
} 
recent developments in de Broglie-Bohm theory have included various proposals for describing at least large portions of the standard model of particle theory (see e.g. Colin and Struyve 2007, Dürr et al. 2005, Struywe and Westman 2007, and the review in Struyve 2011). Critics, however, argue that the configuration variables in these models (which are guided by the relevant wavefunctional) are not necessarily decohering variables (Wallace 2008). It may well be, as argued in particular in the 'minimalist' model of Struyve and Westman (2007), that there are choices for configuration variables that will ensure that measurement results (suitably construed) will always be well-defined. But should the critics prove correct, the 'measured' classical trajectories will be no more real than those of the minimal interpretation, and pilot-wave theorists would still lack a fully satisfactory solution to the problem of the classical regime.

Much progress has been achieved in recent years on the resolution of these two problems, and generally in the philosophy and foundations of quantum mechanics. One should expect to see more in years to come.

\section{References}

Albert, D. (1992), Quantum Mechanics and Experience (Cambridge, Mass.: Harvard University Press).

Allori, V., and Zanghì, N. (2009), 'On the Classical Limit of Quantum Mechanics', Foundations of Physics 39(1), 20-32.

Bacciagaluppi, G. (2000), 'Delocalised Properties in the Modal Interpretation of a Continuous Model of Decoherence', Foundations of Physics 30, $1431-1444$.

Bacciagaluppi, G. (2003), 'The Role of Decoherence in Quantum Mechanics', in E. N. Zalta (ed.), The Stanford Encyclopedia of Philosophy (Fall 2008 edition, http://plato.stanford.edu/archives/fall2008/entries/qm-decoherence/).

Bacciagaluppi, G. (2008), 'The Statistical Interpretation according to Born and Heisenberg', in C. Joas, C. Lehner and J. Renn (eds.), HQ-1: Conference on the History of Quantum Physics (Vols. I and II), MPIWG preprint series, Vol. 350 (Berlin: MPIWG), chapter 14 (vol. II), pp. 269-288 (available at http://www.mpiwg-berlin.mpg.de/en/resources/preprints.html). 
Bacciagaluppi, G. (2010), 'Collapse Theories as Beable Theories', Manuscrito 33(1) (special issue edited by D. Krause and O. Bueno), 19-54.

Bacciagaluppi, G., and Crull, E. (2009), 'Heisenberg (and Schrödinger, and Pauli) on Hidden Variables', Studies in History and Philosophy of Modern Physics 40, 374-382.

Bacciagaluppi, G., and Valentini, A. (2009), Quantum Theory at the Crossroads: Reconsidering the 1927 Solvay Conference (Cambridge: Cambridge University Press).

Barbour, J. B. (1999), The End of Time (London: Weidenfeld and Nicolson).

Bassi, A., and Ghirardi, G.C. (2000), 'A General Argument against the Universal Validity of the Superposition Principle', Physics Letters A 275, 373-381.

Bell, J. S. (1966), 'On the Problem of Hidden Variables in Quantum Mechanics', Reviews of Modern Physics 38, 447-452. Reprinted in Speakable and Unspeakable in Quantum Mechanics (Cambridge: Cambridge University Press), pp. 1-13.

Bohr, N. (1949), 'Discussion with Einstein on Epistemological Problems in Atomic Physics', in P. A. Schilpp (ed.), Albert Einstein: PhilosopherScientist, The Library of Living Philosophers, vol. VII (La Salle: Open Court), pp. 201-241.

Born, M. (ed.) (1969), Albert Einstein, Hedwig und Max Born: Briefwechsel 1916-1955 (München: Nymphenburger Verlagshandlung). Translated by I. Born as The Born-Einstein letters: Correspondence between Albert Einstein and Max and Hedwig Born from 1916-1955 (London: Macmillan, 1971).

Born, M., and Heisenberg, W. (1928), 'La mécanique des quanta [Quantenmechanik]', in Lorentz (1928), pp. 143-184. Translated (from the original German) in Bacciagaluppi and Valentini (2009), pp. 408-447.

Broglie, L. de (1928), 'La nouvelle dynamique des quanta', in Lorentz (1928), pp. 105-141. Translated in Bacciagaluppi and Valentini (2009), pp. 374407. 
Brown, H. R. (1986), 'The Insolubility Proof of the Quantum Measurement Problem', Foundations of Physics 16, 857-870.

Busch, P. (2003), 'Quantum States and Generalized Observables: A Simple Proof of Gleason's Theorem', Physical Review Letters 91, 120403/1-4.

Busch, P., Grabowski, M., and Lahti, P. (1995), Operational Quantum Physics (Berlin and Heidelberg: Springer).

Busch, P., and Shimony, A. (1996), 'Insolubility of the Quantum Measurement Problem for Unsharp Observables', Studies in History and Philosophy of Modern Physics 27B, 397-404.

Cattaneo, G., Marsico, T., Nisticò, G, and Bacciagaluppi, G. (1997), 'A Concrete Procedure for Obtaining Sharp Reconstructions of Unsharp Observables in Finite-Dimensional Quantum Mechanics', Foundations of Physics 27, 1323-1343.

Colin, S., and Struyve, W. (2007), 'A Dirac Sea Pilot-wave Model for Quantum Field Theory', Journal of Physics A 40, 7309-7342.

Deutsch, D. (1999), 'Quantum Theory of Probability and Decisions', Proceedings of the Royal Society of London A 455, 3129-3137.

Donald, M. (1998), 'Discontinuity and Continuity of Definite Properties in the Modal Interpretation', in D. Dieks and P. E. Vermaas (eds.), The Modal Interpretation of Quantum Mechanics (Dordrecht: Kluwer), pp. 213-222.

Dürr, D., Goldstein, S., Tumulka, R., and Zanghì, N. (2005), 'Bell-Type Quantum Field Theories', Journal of Physics A 38, R1-R43.

Einstein, A. (1953), 'Elementare Überlegungen zur Interpretation der Grundlagen der Quanten-Mechanik', in Scientific Papers Presented to Max Born (Edinburgh: Oliver and Boyd), pp. 33-40. Translated by R. Deltete in Nature 356(2 April 1992), 393-395.

Fine, A. (1970), 'Insolubility of the Quantum Measurement Problem', Physical Review D 2, 2783-2787.

Fine, A. (1973), 'Probability and the Interpretation of Quantum Mechanics', British Journal for the Philosophy of Science 24, 1-37. 
Fuchs, C. A. (2010), 'QBism, the Perimeter of Quantum Bayesianism' (http://arxiv.org/abs/1003.5209).

Ghirardi, G.C., Rimini, A., and Weber, T. (1986), 'Unified Dynamics for Microscopic and Macroscopic Systems', Physical Review D 34, 470-491.

Gleason, A. M. (1957), 'Measures on the Closed Subspaces of a Hilbert Space', Journal of Mathematics and Mechanics 6(6), 885-893.

Heisenberg, W. (1927), 'Über den anschaulichen Inhalt der quantentheoretischen Kinematik und Mechanik', Zeitschrift für Physik 43, 172-198.

Heisenberg, W. (1930), Die physikalischen Prinzipien der Quantentheorie. (Leipzig: Hirzel). Translated by C. Eckart and F. C. Hoyt as The Physical Principles of the Quantum Theory (Chicago: University of Chicago Press, 1930).

Heisenberg, W. (1949), Wandlungen in den Grundlagen der Naturwissenschaft (Zürich: Hirzel).

Heisenberg, W. (1985), 'Ist eine deterministische Ergänzung der Quantenmechanik möglich?', in W. Pauli, Wissenschaftlicher Briefwechsel mit Bohr, Einstein, Heisenberg u.a., Band II: 1930-1939, edited by K. v. Meyenn, A. Hermann and V. F. Weisskopf (Berlin and Heidelberg: Springer), pp. 407-418. Translated by E. Crull and G. Bacciagaluppi (with a brief introduction, http://philsci-archive.pitt.edu/8590/).

Hermann, G. (1935), 'Die naturphilosophischen Grundlagen der Quantenmechanik', Abhandlungen der Fries'schen Schule 6, 75-152. Section 7, 'Der Zirkel in Neumanns Beweis', translated by M. Seevinck (http://mpseevinck.ruhosting.nl/seevinck/trans.pdf).

Holland, P. R. (1995), The Quantum Theory of Motion: An Account of the de Broglie-Bohm Causal Interpretation of Quantum Mechanics (Cambridge: Cambridge University Press).

Jauch, J. M., and Piron, C. (1969), 'On the Structure of Quantal Proposition Systems', Helvetica Physica Acta 42, 842-848.

Lewis, P. J. (2010), 'Probability in Everettian Quantum Mechanics', Manuscrito 33(1) (special issue edited by D. Krause and O. Bueno), 285-306. 
Lo, H.-K., and Chau, H. F. (1997), 'Is Quantum Bit Commitment Really Possible?', Physical Review Letters 78, 3410-3413.

Lorentz, H. A. (ed.) (1928), Electrons et photons: rapports et discussions du cinquième conseil de physique Solvay (Paris: Gauthier-Villars),

Mayers, D. (1997), 'Unconditionally Secure Quantum Bit Commitment is Impossible', Physical Review Letters 78, 3414-3417.

Mott, N. F. (1929), 'The Wave Mechanics of $\alpha$-ray Tracks', Proceedings of the Royal Society of London A 126(1930, No. 800 of 2 December 1929), 79-84.

Neumann, J. von (1932), Mathematische Grundlagen der Quantenmechanik (Berlin: Springer). Translated by R. T. Beyer as Mathematical Foundations of Quantum Mechanics (Princeton: Princeton University Press, 1955).

Price, H. (1996), Time's Arrow and Archimedes' Point (New York: Oxford University Press).

Przibram, K. (ed.) (1963), Briefe zur Wellenmechanik (Wien: Springer). Translated by M. J. Klein as Letters on Wave Mechanics (New York: Philosophical Library, 1967).

Saunders, S., Barrett, J., Kent, A., and Wallace, D. (eds.) (2010), Many Worlds? Everett, Quantum Theory, and Reality (Oxford: Oxford University Press).

Schrödinger, E. (1926), 'Der stetige Übergang von der Mikro- zur Makromechanik', Naturwissenschaften 14, 664-666.

Schrödinger, E. (1928), 'Mécanique des ondes [Wellenmechanik]', in Lorentz (1928), pp 185-213. Translated (from the original German) in Bacciagaluppi and Valentini (2009), pp. 448-476.

Schrödinger, E. (1935), 'Die gegenwärtige Situation in der Quantenmechanik', Naturwissenschaften 23, 807-812, 823-828, 844-849. Translated in J. A. Wheeler and W. H. Zurek (eds.), Quantum Theory and Measurement (Princeton: Princeton University Press, 1983), pp. 152-167.

Schulman, L. S. (1997), Time's Arrows and Quantum Measurement (Cambridge: Cambridge University Press). 
Struyve, W. (2011), 'Pilot-wave Approaches to Quantum Field Theory', Journal of Physics: Conference Series 306, 012047/1-10.

Struyve, W., and Westman, H. (2007), 'A Minimalist Pilot-wave Model for Quantum Electrodynamics', Proceedings of the Royal Society of London A 463, 3115-3129.

Wallace, D. (2001), 'Implications of Quantum Theory in the Foundations of Statistical Mechanics' (http://philsci-archive.pitt.edu/410/).

Wallace, D. (2007), 'Quantum Probability from Subjective Likelihood: Improving on Deutsch's Proof of the Probability Rule', Studies in History and Philosophy of Modern Physics 38, 311-332.

Wallace, D. (2008), 'Philosophy of Quantum Mechanics', in D. Rickles (ed.), The Ashgate Companion to Contemporary Philosophy of Physics (Aldershot: Ashgate), pp. 16-98. (Preliminary version available as 'The Quantum Measurement Problem: State of Play (December 2007)', http://philsciarchive.pitt.edu/3420/).

Zurek, W. H. (2003), 'Decoherence, Einselection, and the Quantum Origins of the Classical', Reviews of Modern Physics 75, 715-775.

Zurek, W. H., and Paz, J.-P. (1994), 'Decoherence, Chaos, and the Second Law', Physical Review Letters 72, 2508-2511. 